IJMMS 2003:1, 27-63

PII. S016117120301233X

http://ijmms.hindawi.com

(c) Hindawi Publishing Corp.

\title{
MULTILOCAL INVARIANTS FOR THE CLASSICAL GROUPS
}

\author{
PAUL F. DHOOGHE
}

Received 11 March 2001

\begin{abstract}
Multilocal higher-order invariants, which are higher-order invariants defined at distinct points of representation space, for the classical groups are derived in a systematic way. The basic invariants for the classical groups are the well-known polynomial or rational invariants as derived from the Capelli identities. Higherorder invariants are then constructed from the former ones by means of total derivatives. At each order, it appears that the invariants obtained in this way do not generate all invariants. The necessary additional invariants are constructed from the invariant polynomials on the Lie algebra of the Lie transformation groups.
\end{abstract}

2000 Mathematics Subject Classification: 53A20, 53A55, 57S25.

1. Introduction. The study of invariants stands in contrast with the more structural approaches in differential geometry as, for example, formulated in the books of Kobayashi and Nomizu [13]. One of the main reasons for this is that within this structural approach, invariants appear with a precise geometrical meaning inherited from the structure. When not considered in this context, invariants are, by definition, members of invariant rings of functions which, if certain regularity conditions are imposed, are nothing else but rings generated by a set of defining coordinates of the orbits of the group action on a given manifold. Moreover, when considered as member of the coordinate ring of the orbits, subspaces of a jet bundle, a constant function is not necessarily an absolute invariant. The function may depend on the coordinates with respect to which the partial derivatives are calculated and hence is not necessarily invariant under general reparameterization. For this reason the distinction is made between relative and absolute invariants [26]. But, in geometry, we are, in particular, interested in invariants of a certain weight, which then determine invariant symmetric covariant tensorfields, which are coordinate independent quantities.

We start with a good reason to undertake the study of invariants. Consider the following well-known problem: "Given the action of a group $G$ on a manifold $M$ and two sets of points in $M$, when does an element exist in $G$ which transforms the first set into the second one?" This is the classical problem of invariants. We prefer to tackle the question in a more general form. Consider two sets of points in $M$ and/or tangent vectors and/or higher-order tangent vectors at a distinct points. Say $\mathscr{S}_{1}=\left\{\xi_{i}, \ldots,\left.X\right|_{\xi_{j}}, \ldots,\left.Y Y \ldots Y\right|_{\xi_{k}}, \ldots\right\}$ 
and $\mathscr{S}_{2}=\left\{\eta_{i}, \ldots,\left.X\right|_{\eta_{j}}, \ldots,\left.Y Y \ldots Y\right|_{\eta_{k}}, \ldots\right\}$. The question then is when does there exist an element $g \in G$ such that $g\left(\mathscr{S}_{1}\right)=\mathscr{S}_{2}$ and what are the general criteria? The problem, though interesting in itself, appears in problems related to the existence of invariant kernels [7] and partial differential equations and, in particular, is raised among others in computer vision [3, 16, 20, 24] and in numerical algorithms related to symmetry [18].

One way to answer this type of question is to study invariants for the extended action of $G$ on higher-order tangent vectors located at distinct points of the manifold. Such invariants will be called multilocal differential invariants because they are defined at distinct points of the manifold and also depend on the higher-order differential structure of curves in $M$ through the distinct points. The natural setting for these problems seems to be multispace and multijet space. Such invariants appear under different names in the literature, in particular, as semidifferential invariants [24] and as joint invariants [19].

In this paper, we restrict attention to the local problem and to invariants which are typically curve invariants. This implies that the invariants are meant to be evaluated locally along curves. The development of the same ideas to submanifolds is not considered here. Nonetheless, it turns out that the invariants yielding the key information are tensors on jet bundles and hence, the results are suitable for generalization to the theory of submanifolds.

For each of the groups, we start with "point invariants," namely, invariant functions defined on the zero-order jet space over the manifold. For the linear transformation groups on $\mathbb{R}^{n}$, these invariants are derived from the Capelli identities and are found in the classical literature on the subject [9, 25]. The point invariants are, as one expects, orbit invariants on multispace, which is an $r$-fold product of the representation space with itself. Invariants, depending on higher-order tangent vectors, are then defined on appropriate jet bundles over these products.

The simplest example is given by the standard action of $\mathrm{SO}(3)$ on $V=\mathbb{E}^{3}$. Let $\mathscr{E}_{2}=V_{1} \times V_{2}$ be the multispace. With $x$ as variable on the first factor and $y$ as variable on the second, the functions $2(x, x), 2(x, y)$, and $2(y, y)$ are the well-known invariants of this action. 2 stands for the Euclidean quadratic form on $V$. The rank of multispace equals 2, which is the smallest number of factors needed for the orbits to have maximal dimension. Taking the firstorder jet bundle over the first factor gives the space $J^{1} V_{1} \times V_{2}$. The invariants, together with their total derivatives, yield the set $\{2(x, x), 2(x, y), 2(y, y)$, $2(\dot{x}, x), 2(\dot{x}, y)\}$. The set thus obtained is not maximal, one lacks an invariant function in order to have a complete set. The missing function is the wellknown Euclidean metric function on the first-order jet bundle, namely $2(\dot{x}, \dot{x})$. There are several ways to derive this function. (1) Because the action is linear and extends linearly to the tangent space, we may reapply the Capelli identities to the variables $(x, y, \dot{x})$. (2) We may construct the space $J^{1} V_{1} \times V_{2} \times J^{1} V_{3}$ equipped with the variables $x, \dot{x}, y, z, \dot{z}$. The total derivative with respect to the 
third factor of the invariant $2(\dot{x}, z)$ yields $2(\dot{x}, \dot{z})$. The equivariant embedding $\jmath: J^{1} V_{1} \times V_{2} \rightarrow J^{1} V_{1} \times V_{2} \times J^{1} V_{3}$ given by $z=x, \dot{z}=\dot{x}$ then yields the desired invariant namely $\jmath^{*} 2(\dot{x}, \dot{z})$. (3) Because the orbits of $\mathrm{SO}(3)$ are diffeomorphic to the group, they carry the invariant metric determined by the translation of the Killing form on the orbits. An invariant Riemannian metric on $\mathscr{E}_{2}$ is found by means of the construction of an invariant bundle transversal to the orbits. The metric results from the Killing form on the orbits and the choice of an Euclidean metric in the transversal bundle which is taken orthogonal to the orbits. Consider the generating vector fields

$$
\begin{aligned}
& Z_{1}=x_{1} \partial_{x_{2}}-x_{2} \partial_{x_{1}}+y_{1} \partial_{y_{2}}-y_{2} \partial_{y_{1}}, \\
& Z_{2}=x_{1} \partial_{x_{3}}-x_{3} \partial_{x_{1}}+y_{1} \partial_{y_{3}}-y_{3} \partial_{y_{1}}, \\
& Z_{3}=x_{3} \partial_{x_{2}}-x_{2} \partial_{x_{3}}+y_{3} \partial_{y_{2}}-y_{2} \partial_{y_{3}}
\end{aligned}
$$

and the invariant transversal vector fields

$$
\begin{gathered}
Z_{4}=x_{1} \partial_{x_{1}}+x_{2} \partial_{x_{2}}+x_{3} \partial_{x_{3}}, \quad Z_{5}=y_{1} \partial_{y_{1}}+y_{2} \partial_{y_{2}}+y_{3} \partial_{y_{3}}, \\
Z_{4}=-y_{1} \partial_{x_{1}}-y_{2} \partial_{x_{2}}-y_{3} \partial_{x_{3}}+x_{1} \partial_{y_{1}}+x_{2} \partial_{y_{2}}+x_{3} \partial_{y_{3}} .
\end{gathered}
$$

Defining these vector fields as orthonormal determines an invariant metric. This metric, taken at the invariant subspace $y=0$, restricted to the tangent bundle of the first component of the multispace $\mathscr{E}_{2}$, and considered as function on $J^{1} V$, is given by $\phi=\left(x_{1}^{2}+x_{2}^{2}+x_{3}^{2}\right)\left[\left(\dot{x}_{1}\right)^{2}+\left(\dot{x}_{1}\right)^{2}+\left(\dot{x}_{1}\right)^{2}\right]$. Because $\left(x_{1}^{2}+\right.$ $\left.x_{2}^{2}+x_{3}^{2}\right)$ is invariant, we find the extra invariant $2(\dot{x}, \dot{x})$. In this paper, we show that this happens at each level for all the classical groups. The same approach is then applied.

The results of the present paper can be summarized as follows. On the firstorder bundle, we find, besides the zero-order invariants and their first-order prolongations, an additional set of functionally independent invariants which, together with the former ones, generate the sheaf of invariant functions. We show that, if formulated properly, these additional invariants are related to the invariant polynomes on the Lie algebra of the transformation group. Hence, they are symmetric tensors on the orbits of the group in the zero-order jet bundle. In this sense, they relate directly to the geometry of the orbits of the group in zero-order space. The same procedure then repeats at each order. The dimension of the space upon which the group acts is crucial at each step. For each of the groups, we follow the same procedure and present some aspects of the geometry involved.

The following transformation groups are treated: (1) the Euclidean group, $\operatorname{Eucl}(n)$; (2) the similarity group, $\operatorname{Sim}(n)$; (3) the symplectic group, $\operatorname{Sym}(n)$; (4) the volume-preserving group, $\operatorname{Vol}(n)$; (5) the affine group, $\operatorname{Aff}(n)$; (6) the projective group, $\mathrm{Pl}(n)$; and (7) the conformal group, $\mathrm{CO}^{(1)}(n)$. In each case, we only consider the standard action. 
For each of the groups from (1) to (5), we examine the action of their linear part and construct the invariant sheaf. Then, by means of a homogenization procedure, the sheaf of invariants for the transitive groups is found. Remark that the following groups are semisimple: $\mathrm{SO}(n), O(n), \mathrm{Sp}(n), \mathrm{Sl}(n), \mathrm{Pl}(n)$, and $\mathrm{CO}^{(1)}(n)$. Furthermore, the results for $\mathrm{CO}(n)$ are derived from $\mathrm{SO}(n)$ and those for $\mathrm{Gl}(n)$ from $\mathrm{Sl}(n)$ by projectivization of a set of generators of the invariant sheaf.

In the first section, we review the basic results on invariants for the classical linear groups such as given in the classical book of Weyl on the subject [25] or more recently by Fulton and Harris [9]. The subsequent sections then treat the higher-order multilocal invariants for the different groups. The Capelli construction of the invariants is a well-debated subject, and we refer to the literature [12, 23]. A different approach has been given by Olver [17, 19], and Fels [8] in terms of moving coframes. The construction of the invariant metric in multispace, which we use here, is very close to this approach, although for the point invariants, we use the classical theorems.

Besides the relative invariants, which depend on the parameterization of the curves, we have to find a parameterization which is invariant under the induced action of the group. Such parameters are called $G$-invariant parameters [16]. Clearly, the construction of such parameters is not unique; there are several types of possible parameters. For example, the parameter may be determined by the integral of the $k$ th root of an invariant function of weight $k$, determined on the jet bundle. But we may also determine a parameter by means of the normalization of the prolonged curve with respect to an invariant symmetric tensor field on the jet bundle. For example, in the Euclidean case, there exists a tensor field which lives on the zero-order jet bundle determining the Euclidean arc length, but, for example, in the case of a curve in one projective space, we will show that the Schwarzian derivative, which determines a projective parameter, is nothing but a normalization with respect to the Killing form on the orbit of the projective group in the second-order jet bundle. As said in former paragraphs, we will not go into a systematic examination of the existence of such invariant parameters but will give examples of such parameters.

The general setting for this paper is the $C^{\infty}$-category of manifolds and functions. Restriction to polynomials or rational functions would be more advisable in most cases, but, for our interest, the distinction is not very important. The language of sheaf theory is used, which makes the formulation easier and more elegant, but, as the reader will notice, we could also do without it at this stage. The sheafs here are sheafs of germs of $C^{\infty}$-real valued functions, which are fine sheafs, and their global analysis is outside the scope of this paper. For the Lie groups and their algebras used in this paper, we refer to Helgason [11].

2. Elements of the classical theory. Let $V$ be the standard representation space of $\operatorname{Gl}(n)$ and $G$ a subgroup of $\operatorname{Gl}(n)$. Let $\left(e_{i}\right)$ be a basis on $V$; we denote 
by $\left(\xi^{i}\right)$ the corresponding coordinates and use $\xi=\left(\xi^{i}\right)$ to indicate a point in $V$. Classical invariant theory aims at constructing all polynomials $P\left(\xi_{1}, \xi_{2}, \ldots, \xi_{m}\right)$ in $m$ variables on $V$ which are invariant under the action of $G$.

It is indicated to define these polynomials on the product space $\mathscr{E}_{r}=\prod_{\alpha=1}^{r} V_{\alpha}$, where each $V_{\alpha}$ is a copy of $V$. The action of the group $G$ on $\mathscr{E}_{r}$ is the product action. Invariant polynomes or invariant functions in $m$ variables are polynomes or functions on $\mathscr{E}_{r}$, depending on $m$ factors, with $r \geq m$.

Results on the invariants for the classical linear subgroups of $\mathrm{Gl}(n)$ are consequences of the identities of Capelli $[9,25]$. We summarize the results in the form of a theorem.

THEOREM 2.1 [9]. (1) The only polynomial invariants for $\mathrm{Gl}(n)$ and $\mathrm{CO}(n)$ are the constants.

(2) Polynomial invariants $P\left(\xi_{1}, \xi_{2}, \ldots, \xi_{m}\right)$ for $\operatorname{SI}(n)$ can be written as polynomials in the determinants $\left|\xi_{\alpha_{1}} \xi_{\alpha_{2}} \cdots \xi_{\alpha_{n}}\right|, 1 \leq \alpha_{1}<\alpha_{2}<\cdots<\alpha_{n} \leq m$.

(3) Polynomial invariants $P\left(\xi_{1}, \xi_{2}, \ldots, \xi_{m}\right)$ for $\operatorname{Sp}(n)$ (resp., $O(n)$ ) can be written as polynomials in the functions $2\left(\xi_{\alpha}, \xi_{\beta}\right), 1 \leq \alpha<\beta \leq m$, (resp., $1 \leq \alpha \leq \alpha \leq$ $m)$, with 2 the quadratic form which determines the standard action of $\operatorname{Sp}(n)$ (resp., $O(n)$ ) on $V$.

(4) Polynomial invariants $P\left(\xi_{1}, \xi_{2}, \ldots, \xi_{m}\right)$ for $\mathrm{SO}(n)$ can be written as polynomials in the functions $2\left(\xi_{\alpha}, \xi_{\beta}\right)$ and $\left|\xi_{\alpha_{1}} \xi_{\alpha_{2}} \cdots \xi_{\alpha_{n}}\right|$, with $1 \leq \alpha \leq \beta \leq m$ and $1 \leq \alpha_{1}<\alpha_{2}<\cdots<\alpha_{n} \leq m$.

From this theorem, we easily derive rational invariants in $m$ variables for $\mathrm{CO}(n)$ and $\mathrm{Gl}(n)$. The following theorems state the results.

THEOREM 2.2. All rational functions in $\left|\xi_{\alpha_{1}} \xi_{\alpha_{2}} \cdots \xi_{\alpha_{n}}\right| /\left|\xi_{\beta_{1}} \xi_{\beta_{2}} \cdots \xi_{\beta_{n}}\right|$, with $1 \leq \alpha_{1}<\alpha_{2}<\cdots<\alpha_{n} \leq m$ and $1 \leq \beta_{1}<\beta_{2}<\cdots<\beta_{n} \leq m$, are invariants for the standard action of $\mathrm{Gl}(n)$ on $V$.

THEOREM 2.3. All rational functions in $Q\left(\xi_{\alpha_{1}}, \xi_{\alpha_{2}}\right) Q\left(\xi_{\beta_{1}}, \xi_{\beta_{2}}\right)$, with $1 \leq \alpha_{1} \leq$ $\alpha_{2} \leq m$ and $1 \leq \beta_{1} \leq \beta_{2} \leq m$, are invariants for the standard action of $\mathrm{CO}(n)$ on $V$.

The projective group $\mathrm{Pl}(n) \simeq \mathrm{Gl}(n+1) / \pm e^{\lambda}$ Id and the conformal group $\mathrm{CO}^{(1)}(n)$ do not act linearly on $V$ and hence need a different treatment.

First, consider the projective group. Let $\xi_{\alpha_{1}}, \xi_{\alpha_{2}}, \ldots, \xi_{\alpha_{m}}$ be $m$ variables on $V$ and $\xi_{k}$ an extra variable on $V$. We then define

$$
\left|\xi_{\alpha_{1}} \xi_{\alpha_{2}} \cdots \xi_{\alpha_{n}} ; \xi_{k}\right|=\left|\left(\xi_{\alpha_{1}}-\xi_{k}\right)\left(\xi_{\alpha_{2}}-\xi_{k}\right) \cdots\left(\xi_{\alpha_{n}}-\xi_{k}\right)\right|
$$

THEOREM 2.4. All rational functions in

$$
\Pi\left|\xi_{\alpha_{1}} \xi_{\alpha_{2}} \cdots \xi_{\alpha_{n}} ; \xi_{k}\right| / \Pi\left|\xi_{\beta_{1}} \xi_{\beta_{2}} \cdots \xi_{\beta_{n}} ; \xi_{k}\right|
$$

with $1 \leq \alpha_{1}<\alpha_{2}<\cdots<\alpha_{n} \leq m$ and $1 \leq \beta_{1}<\beta_{2}<\cdots<\beta_{n} \leq m$, where the products are taken such that for each $\alpha_{r}$ the number of factors containing the 
variable $\xi_{\alpha_{r}}$ in the nominator equals the number of factors containing $\xi_{\alpha_{r}}$ in the denominator, are invariant for the standard action of $\mathrm{Pl}(n)$ on $V$.

PROoF. The above expression is clearly invariant for translations and the action of the general linear group. For this, we first remark that, because they are invariant under translations, we may put $\xi_{k}=0$. Invariance under the special projective transformations $\tilde{\xi}^{j}=\xi^{j} / 1+a_{i} \xi^{i}$ is easily checked, which proves the proposition.

The group of conformal transformations $\mathrm{CO}^{(1)}(n)$ is the first prolongation of the group $\mathrm{CO}(n)[10,22]$. The group is generated by the elements of the similarity group $\operatorname{Sim}(n)$ and the inversions on the $n$ sphere. As transformation on $V$, the conformal group is generated by the similarity group $\operatorname{Sim}(n)$, together with the special conformal transformations [1]

$$
\tilde{\xi}^{j}=\frac{\xi^{j}-c^{j} 2(\xi, \xi)}{f_{\xi}},
$$

with $f_{\xi}=1-22(c, \xi)+2(c, c) 2(\xi, \xi), c \in V$.

THEOREM 2.5. Let $\left(\xi_{\alpha_{i}}\right)$ be $m$ variables on $V$ and $d$ the distance function defined by the quadratic form 2. All rational functions in $\Pi d\left(\xi_{\alpha_{i}} \xi_{\alpha_{j}}\right) / \Pi d\left(\xi_{\beta_{i}} \xi_{\beta_{j}}\right)$, with $1 \leq \alpha_{i}<\alpha_{j} \leq m$ and $1 \leq \beta_{i}<\beta_{j} \leq m$ and where the product is taken such that for each $\alpha_{r}$ the degree of the variable $\xi_{\alpha_{r}}$ in the nominator equals the degree of this variable in the denominator, are invariant for the standard action of $\mathrm{CO}^{(1)}(n)$ on $V$.

Proof. The expressions in the theorem are clearly invariant for the action of the translations and the group $\mathrm{CO}(n)$. We only have to prove invariance for the action of the special transformations (2.3). The proof then follows from the application of the following lemma to the above functions.

LEMMA 2.6 [1]. Let $\xi_{1}, \xi_{2}$ be two points in $V$. Then,

$$
d\left(\tilde{\xi}_{1}, \tilde{\xi}_{2}\right)=\frac{d\left(\xi_{1}, \xi_{2}\right)}{f_{\xi_{1}} f_{\xi_{2}}}
$$

3. Multispace. Let $V$ be as before and $\mathscr{E}=\prod_{\alpha=1}^{r} V_{\alpha}$, the product of $r$ copies of $V . \mathscr{E}$ is called multispace constructed upon $V$. We denote by $\left(\xi_{\alpha}^{i}\right)$ the coordinates on the $\alpha$ th copy in the product and call $V_{\alpha}$ the $\alpha$-layer of $\mathscr{E}$. The projection $\rho_{\alpha}: \mathscr{E} \rightarrow V_{\alpha}$ is the mapping onto the $\alpha$ th component. The identification of $V$ with a specific layer is called the layer mapping, which is given by $i_{\alpha}: V \rightarrow V_{\alpha}$, and the image projection $\pi$ is defined as the superposition of the different layers upon $V$. In other words, $\pi$ is the $r$-valued mapping defined by the set of $r$ functions $\left(\rho_{\alpha}\right)$ of $\mathscr{E}$ followed by the natural identification of each layer with $V$. Remark that $\pi o i_{\alpha}=$ Id for each $\alpha$. 
The order of multispace $\mathscr{E}$ equals the number of layers of $\mathscr{E}$. When necessary, we indicate the order of multispace explicitly and use $\mathscr{E}_{r}$.

Calling $\triangle_{k l}: \xi_{k}=\xi_{l}$ a diagonal of $\mathscr{E}_{r}$, we define the following operations: (1) the permutation mapping of two layers $s_{k l}: \mathscr{E} \rightarrow \mathscr{E}$, which permutes the layers $V_{k}$ and $V_{l}$ and (2) the diagonal mapping $\jmath_{k l}: \mathscr{E}_{r-1} \rightarrow \mathscr{E}_{r}$, which is the injection defined by $\left(\xi_{\alpha}\right) \mapsto\left(\xi_{1}, \ldots, \xi_{k}, \ldots, \xi_{k}, \ldots, \xi_{r-1}\right)$ identifying $\mathscr{E}_{r-1}$ with the diagonal $\triangle k l$.

Any multispace possesses, in a natural way, sets of commuting vector fields which are useful in the construction of invariant. The following lemma is one example.

LEMMA 3.1. Let $\left(\xi_{\alpha}^{i}\right), \alpha=1, \ldots, r, i=1, \ldots, n$, be coordinates on $\mathscr{E}_{r}$. Then, for all $k, l, \alpha, \beta$

$$
\left[\sum_{i} \xi_{\alpha}^{i} \partial_{\xi_{\beta}^{i}}, \sum_{\gamma} \xi_{\gamma}^{k} \partial_{\xi_{\gamma}^{l}}\right]=0
$$

Proof. A simple calculation gives

$$
\left[\sum_{i} \xi_{\alpha}^{i} \partial_{\xi_{\beta}^{i}}, \sum_{\gamma} \xi_{\gamma}^{k} \partial_{\xi_{\gamma}^{l}}\right]=\xi_{\alpha}^{i} \delta_{k}^{i} \delta_{\gamma}^{\beta} \partial_{\xi_{\gamma}^{l}}-\xi_{\gamma}^{k} \delta_{l}^{i} \delta_{\alpha}^{\gamma} \partial_{\xi_{\beta}^{i}}=0
$$

We call a point $\xi$ in multispace $\mathscr{E}_{r}$ generic if the image projection $\pi(\xi)$ of $\xi$ consists of $r$ distinct points in $V$. Let $\xi^{o}$ be a point in $V$, then there exists a canonical lift to $\mathscr{E}_{r}$ which consists of the unique point $\widehat{\xi^{o}}=\left(\xi^{o}, \xi^{o}, \ldots, \xi^{o}\right)$. We call such a point $\widehat{\xi^{o}} \in \mathscr{E}_{r}$ diagonal or canonical.

Remark that an arbitrary curve in $\mathscr{E}_{r}$ describes the evolution of $r$ points in $V$. A generic curve in $\mathscr{E}$ is, by definition, a curve containing a dense subset of generic points. A generic curve is locally projected upon $r$ distinct curves in $V$ by the image map. On the other hand, if a curve in $V$ is given, many different lifts to $\mathscr{E}_{r}$ are possible. First of all, if $\xi=\gamma(t)$ is a curve in $V$, then it is easy to see that there exists a unique curve $\hat{\gamma}(t)$ in $\mathscr{E}_{r}$ such that for each $\alpha$, $\rho_{\alpha}(\hat{\gamma}(t))=\hat{\gamma}_{\alpha}(t)=\gamma(t)$. We call such curves diagonal or canonical. It describes the evolution of $r$ coinciding points in $V$.

Another type of curve in $\mathscr{E}_{r}$ is defined as follows. Let $\xi=\gamma(t)$ be a curve in $V$ and $\hat{\xi}=\left\{\xi_{1}, \xi_{2}, \ldots, \xi_{r}\right\}$ be $r$ points in $V$ such that for a given $\alpha, \xi_{\alpha} \in \gamma(t)$. Then, $\hat{\gamma}(t): t \mapsto\left(\xi_{1}, \xi_{2}, \ldots, \xi_{\alpha}=\gamma(t), \ldots, \xi_{r}\right)$ defines a curve in $\mathscr{E}_{r}$. We call this curve a layer curve and $\xi_{\alpha}$ the running point. Remark that the other points $\xi_{i}, i \neq \alpha$, may or may not belong to $\gamma$. With $\pi$, the image projection, we have $\pi(\hat{\gamma}(t))=\gamma(t) \cup_{i \neq \alpha}^{r}\left\{\pi\left(\xi_{i}\right)\right\}$. In particular, a layer curve is the lift of a curve $\gamma(t)$ in $V$ if all points $\xi_{\alpha} ; 1, \ldots, r$ belong to $\gamma(t)$.

In general, we would like to have a parameterization of the layer curve different from the one of the original curve in $V$. This brings us to the following definition. 
DEFINITION 3.2. Let $\gamma(t)$ be a curve in $V$ and $\left(\xi_{i}\right)$ a set of $r$ points in $V$, all belonging to $\gamma(t)$. Then, an $\alpha$-layer curve $\hat{\gamma}$, associated with $\gamma(t)$, is a curve $\hat{\gamma}(t): t \mapsto\left(\xi_{1}, \xi_{2}, \ldots, \xi_{\alpha}=\gamma(\phi(t)), \ldots, \xi_{r}\right)$, where $\phi$ is a reparameterization of $\gamma$.

The condition that $\hat{\gamma}$ is a layer curve associated with $\gamma(t)$ in the $\alpha$ th layer can be reformulated as $\operatorname{Im} \rho_{\alpha}(\hat{\gamma})=\operatorname{Im}(\gamma)$ and $\rho_{\beta}(\hat{\gamma})=\xi_{\beta}$ for $\beta \neq \alpha$.

The construction of a general lifting of a curve is, in fact, a kind of superposition of $r$ layer curves. Hence, we have the following definition.

DEFINITION 3.3. Let $\gamma(t)$ be a curve in $V$. Then, $\hat{\gamma}(t)$ in $\mathscr{E}$ is a lift of $\gamma(t)$ if the image of the image projection of $\hat{\gamma}(t)$ equals the image of $\gamma(t)$.

Let $\left(\xi_{\alpha}^{i}\right)$ be the coordinates on $V_{\alpha}$ and $\xi^{i}=\gamma^{i}(t)$ a curve in $V$. A lifted curve on $\mathscr{E}$ is given by

$$
\xi_{\alpha}^{i}=\hat{\gamma}_{\alpha}^{i}=i_{\alpha}\left(\gamma\left(\phi_{\alpha}(t)\right)\right),
$$

where $\phi$ is a reparameterization in the domain of the curve $\gamma$. We remark that parameterization may differ from layer to layer. Hence the lift, as a parameterized curve, is not unique; indeed, we accept the freedom to move the distinct points with a different speed along the curve. Hence, keeping the $r-1$ points $\xi_{\beta}$, with $\beta \neq \alpha$ for a given $\alpha$, fixed and $\xi_{\alpha}$ as the running point determines a layer curve associated with $\gamma(t)$.

4. Jets of curves in multispace. Consider the set of germs of curves in $V$ and denote this space by $\mathbf{C}(V)$. Recall that germ equivalence of two curves is defined as $\gamma_{1}(t) \sim t^{o} \gamma_{2}(t)$ if and only if both curves coincide on a neighborhood of $t^{o}$. The set of germs is the sheaf of germ equivalent curves on $\mathbb{R}$ with values in $V$.

Jet equivalence is then introduced as an equivalence on $\mathbf{C}(V)$. Let $\gamma_{1}(t)$ and $\gamma_{2}(t)$ be the representatives of two germs in $\mathbf{C}(V)$, both are $k$ jet equivalent if their derivatives up to order $k$ coincide at $t$. This equivalence clearly does not depend upon the choice of the representatives. We generally indicate a germ by a representative and the $k$ jet of a curve germ by $j_{t}^{k}(\gamma)$. It is assumed that the germ is taken at the point $t$. The set of $k$ jets of germs of curves in $V$ is the space $J^{k}(\mathbb{R}, V)$. The set of infinite jets $J(\mathbb{R} V)$, is defined as the inverse limit of the sequence $\left(J^{k}(\mathbb{R}, V)\right)_{k}$. This space admits a ring of $C^{\infty}$-functions $\mathscr{F}(J(\mathbb{R}, V))$, defined by the direct limit of the sets $\left\{\mathscr{F}\left(J^{k}(\mathbb{R}, V)\right)\right\}$ with respect to the projections $\pi_{k}: J(\mathbb{R}, V) \rightarrow J^{k}(\mathbb{R}, V)$. In the sequel, we consider each set $\mathscr{F}\left(J^{k}(\mathbb{R}, V)\right)$ as a natural subset of $\mathscr{F}(J(\mathbb{R}, V))$. A section $\sigma: \mathbb{R} \rightarrow J(\mathbb{R}, V)$ is called integrable if $\sigma$ equals the jet of a curve at each of its source points.

Let $\alpha: J^{k}(\mathbb{R}, V) \rightarrow \mathbb{R}$ be the source map defined as $j_{t}^{k} \gamma \mapsto t$. The fibre $\alpha^{-1}(0)$ is a bundle over the target space $V$. We call this bundle $J^{k}(V)$. This bundle is equipped with the projection map $\beta: J^{k}(V) \rightarrow V$ by $j_{0}^{k}(\gamma) \mapsto \gamma(0)$. We denote 
elements in $J^{k}(V)$ by $j^{k}(\gamma)$. The bundle of infinite jets is denoted by $J(V)$. Again, we call a curve $\sigma: \mathbb{R} \rightarrow J(V)$ integrable if it is the infinite jet of a curve in $V$ at each of the points of $\beta(\sigma(t))$.

Let $\partial_{t}$ be the derivative operator on $\mathbb{R}$; the total lift of this operator, $\partial_{t}^{h}$, as an operator on $\mathscr{F}(J(\mathbb{R}, V))$, is given by $\partial_{t} f(\sigma(t))=\left(\partial_{t}^{h} f\right)(\sigma(t))$ for $f$ in $\mathscr{F}(J(\mathbb{R}, V))$ and $\sigma$ integrable section: $\sigma: \mathbb{R} \rightarrow J(\mathbb{R}, V)$.

Let $X \in \mathscr{L}(V)$ be a vector field with flow $\phi_{t}$. Because $\phi_{t}$ is a local diffeomorphism on $V$, composition with curve mappings defines the prolonged action of the local diffeomorphism on the jet space $J^{k}(V)$. Let $\gamma(s)$ be a curve, then $\phi_{t}(\gamma(s))$ is the composition with the local diffeomorphism for a fixed $t$ and the action on $J^{k}(V)$ is given by $\phi_{t}^{(k) *} j^{k} \gamma=j^{k}(\phi(\gamma))$. This determines what is called the complete lift $X^{(k)}$, generator of $\phi^{(k)}$, on $J^{k}(V)$. The following proposition, which is well known in jet bundle theory [5, 14, 15], gives a constructive definition.

Definition 4.1. Let $X$ be a vector field on $V$. Then, the complete lift $X^{(k)}$ is the unique vector field on $J^{k}(V)$ satisfying the conditions (1) $\left[\partial_{t}^{h}, X^{(k)}\right]=0$ and (2) $\beta_{*} X^{(k)}=X$.

The above definitions extend in a natural way to multijet space. Let $\gamma(t)$ be a curve in $V$ and $\hat{\gamma}_{\alpha}(t)$ an $\alpha$-layer curve corresponding to $\gamma(t)$ in $\mathscr{E}_{r}$. The 1 jet of $\hat{\gamma}_{\alpha}(t)$ defines a curve in $V_{1} \times V_{2} \times \cdots \times J^{1} V_{\alpha} \times \cdots \times V_{r}$.

This operation is also defined on general curves $\hat{\gamma}$ in $\mathscr{E}_{r}$. The jet extension is applied to the $\alpha$ th component of the curve. It means that we consider the curve as a layer curve keeping all other components fixed. Moreover, we may extend each component $\hat{\gamma}_{\alpha}$ to a specific jet order $k_{\alpha}$. This brings us to products of jet bundles over $V$, which is a bundle over the base manifold $\mathscr{E}_{r}$. This space takes the form of the product

$$
\mathscr{F}^{\left(k_{1}, \ldots, k_{r}\right)}\left(\mathscr{E}_{r}\right)=\prod_{\alpha=1}^{r} J^{k_{\alpha}} V_{\alpha}
$$

and is called multijet space. Points in this space are multijets of curves in $V$. The component $J^{k_{\alpha}} V_{\alpha}$ is the $\alpha$-layer jet bundle.

Let $\hat{\gamma}(t)$ be a curve in $\mathscr{E}_{r}$, and let $p$ be a generic point on $\hat{\gamma}(t)$. A multijet of $\hat{\gamma}(t)$ at $p$ defines, via the image projection, $r$ points together with tangents up to a given order of the curve $\gamma(t)$ at each of the points.

The bundle $\mathscr{g}^{\left(k_{1}, \ldots, k_{r}\right)}\left(\mathscr{E}_{r}\right)$ is a subbundle of $J^{k}\left(\mathscr{E}_{r}\right)$, where $k=\max \left\{k_{1}, \ldots, k_{r}\right\}$, but the jet bundle operations are defined on each layer jet bundle. A section $\sigma$ of $\mathscr{g}^{\left(k_{1}, \ldots, k_{r}\right)}\left(\mathscr{E}_{r}\right)$ can be written as $\sigma=\left(\sigma_{\alpha}\right)$. The section is integrable if and only if each $\sigma_{\alpha}$ is integrable. Each layer carries a total derivative operator, which we denote by $T_{\alpha}$. Let $p=\left(\sigma_{1}\left(t_{o}\right), \ldots, \sigma_{r}\left(t_{o}\right)\right)$, we then have $T_{\alpha} f(p)=$ $\left(\partial_{t} \sigma_{\alpha}^{*} f\right)\left(\sigma_{1}\left(t_{o}\right), \ldots, \sigma_{\alpha-1}\left(t_{o}\right), t_{o}, \sigma_{\alpha+1}\left(t_{o}\right), \ldots, \sigma_{r}\left(t_{o}\right)\right)$, where $f$ is any function on multijet space. 
The complete lift of a vector field on $\mathscr{E}_{r}$ on a jet bundle over a specific layer $J^{k_{\alpha}}\left(V_{\alpha}\right)$ is defined as in Definition 4.1 where the total derivative is $T_{\alpha}$. Let $\left(\xi_{\alpha}^{\left(l_{\alpha}\right) i}\right)$ be the natural coordinates on $\mathscr{F}^{\left(k_{1}, \ldots, k_{r}\right)}\left(\mathscr{E}_{r}\right)$ and $X=A_{\alpha}^{i} \partial_{\xi_{\alpha}^{i}}$ a vector field on $\mathscr{E}_{r}$, then

$$
\tilde{X}=\sum T_{\alpha}^{\left(l_{\alpha}\right)} A_{\alpha}^{i} \partial_{\xi_{\alpha}\left(l_{\alpha}\right) i}
$$

is the total lift of the vector field.

The operations which have been determined on multispaces extend to multijet spaces. Call $J^{k_{\alpha}} V_{\alpha}$ the jet bundle over the $\alpha$ th layer.

(1) Let the jet bundles over the layers $V_{k}$ and $V_{l}$ be of same order. The permutation mapping $\iota_{k l}: \mathscr{F}(\mathscr{E})_{r} \rightarrow \mathscr{J}(\mathscr{E})_{r}$ is a mapping which permutes the two layers together with their jet bundles $J^{m} V_{k}$ and $J^{m} V_{l}$.

(2) A diagonal in multijet space $\triangle_{k l}$ is defined as follows. Let $J^{m_{k}} V_{k}$ and $J^{m_{l}} V_{l}$ be two jet bundles over different layers in $\mathscr{g}(\mathscr{E})_{r}$. Suppose that $m_{k} \leq m_{l}$. Then, the diagonal subspace is given by $\triangle_{k l}: J^{m_{k}} V_{k}=J^{m_{k}} V_{l}$. At $\triangle_{k l}$, the $m_{k}$ jets over both components are identified. A diagonal mapping is an injection $J_{k l}: \mathscr{F}(\mathscr{E})_{r-1} \rightarrow \mathscr{F}(\mathscr{E})_{r}$ with image $\triangle_{k l}$.

5. Group actions on multispace. Let $G$ be a Lie group acting on $V$, the action being given by $\tau: G \times V \rightarrow V$ as $(g, \xi) \mapsto g \cdot \xi$. The induced action on $\mathscr{E}_{r}$ is given by $\tilde{\tau}:\left(g,\left(\xi_{\alpha}\right)\right) \mapsto\left(g \cdot \xi_{1}, g \cdot \xi_{2}, \ldots, g \cdot \xi_{r}\right)$. Any function $P\left(\xi_{1}, \xi_{2}, \ldots, \xi_{m}\right), m \leq r$, is invariant for the action of $G$ if and only if $P\left(\xi_{1}, \xi_{2}, \ldots, \xi_{m}\right)$ is constant on the orbits of $G$ in $\mathscr{E}_{r}$. We easily verify the following proposition.

Proposition 5.1. The maps $\pi, j_{k l}$, and $s_{k l}$ are equivariant.

Let $\mathfrak{g}$ be the Lie algebra of $G$ and $\left(e_{a}\right), a=1, \ldots, \operatorname{dim} \mathfrak{g}$, a basis. The vector field $\kappa: e_{a} \mapsto X_{a}=\left.D \tau\left(e_{a}\right)\right|_{e}$, with $e$ the identity in $G$, is a fundamental (or generating) vector field. Given the action of $G$ on $\mathscr{E}_{r}$, we define the singular subset $\Sigma_{G}=$ $\left\{\xi \in \mathscr{E}_{r} \mid \mathbf{r k}\left\{X_{a}\right\}<\operatorname{dim} \mathfrak{g}\right\}$, where $X_{a}$ is a complete set of fundamental vector fields. The regular subset is the complement $\mathscr{W}=\mathscr{E}_{r} \backslash \Sigma$. The sheaf of germs of $C^{\infty}$ invariant functions on $\mathcal{W}, \mathscr{A}(\mathcal{W})$, is called the invariant sheaf.

Let $M$ be a manifold and $\mathscr{S}=\left\{f_{1}, f_{2}, \ldots, f_{k}\right\}$ a set of $C^{\infty}$-functions; the set $\mathscr{S}$ is called functionally independent at $p \in M$ if the associated $k$-form $\omega_{\mathscr{S}}=$ $d f_{1} \wedge d f_{2} \wedge \cdots \wedge d f_{k}$ is different from zero at $p$ [20]. The conditions for a set of functions to be functionally independent on a manifold are those of implicit function theorem asserting the existence of a local submanifold at $p$, whose tangent space is the kernel of the set of one forms $\left\{d f_{i}\right\}$. The rank $k$ of $\mathscr{Y}$ equals the codimension of the submanifold.

Because the invariant sheaf is determined by its sections, we only need to give at each point a complete functionally independent set of germs of invariant $C^{\infty}$-functions. Such a set will be called a set of generators. We agree not to mention the passage from functions to germs. To simplify notation, we do 
not mention the base points of the germs either and will give a minimal set of functions needed in the construction of a maximal functionally independent set of sections of the sheaf at each point in the domain of the sheaf. Such a set of generators will be called complete.

Let $X_{a}$ be a fundamental vector field, then $f \in \mathscr{A}(\mathscr{W}) \rightarrow X_{a}(f)=0$, for all $a$. The converse is not always true. It follows that, on a regular subset $\mathcal{W}$, the rank of the sheaf of germs of $C^{\infty}$-invariant functions equals the codimension of the level surface of any set of generators.

Consider the projective equivariant system

$$
\mathscr{E}_{1}=V \stackrel{\pi_{1}^{2}}{\longleftarrow} \mathscr{E}_{2} \stackrel{\pi_{2}^{3}}{\longleftarrow} \mathscr{E}_{3} \leftarrow \cdots \leftarrow \mathscr{E}_{r-1} \stackrel{\pi_{r-1}^{r}}{\longleftarrow} \mathscr{E}_{r} .
$$

Let $\mathscr{O}_{r} \subset \mathscr{E}_{r}$ be an orbit of $G$, then $\pi_{r-1}^{r} \mathcal{O}_{r}$ is an orbit in $\mathscr{E}_{r-1}$. Hence, $\operatorname{dim} \mathscr{O}_{r-1} \leq$ $\operatorname{dim} \hat{O}_{r}$. The orbit dimension is a nondecreasing function on the orbits. Let $\mathscr{Y}$ be the set of maximal orbits. We then define the order of stabilization of the action of $G$ in the limiting system defined by $\mathscr{E}_{r}$, for $r$ large enough, as the rank $k$ such that for all $l>0: \operatorname{dim} \mathscr{O}_{k+l}=\operatorname{dim} \mathscr{O}_{k}$, for orbits in $\mathscr{Y}$.

REMARK 5.2. (1) Let $k$ be the order of stabilization of $G$ in $\mathscr{E}_{r}$. Then, as a consequence of the dimensional requirements, we have $\mathbf{r k} \mathcal{A}\left(\mathscr{W}_{k+1}\right)=\mathbf{r k} \mathcal{A}\left(\mathcal{W}_{k}\right)+n$ $(n=\operatorname{dim}(V))$.

(2) Let $P\left(\xi_{1}, \xi_{2}, \ldots, \xi_{m}\right)$ be a generator in $\mathscr{A}\left(\mathscr{W}_{k}\right)$. Then,

$$
P^{\prime}\left(\xi_{1}, \xi_{2}, \ldots, \xi_{m+1}\right)=s_{m, m+1} P\left(\xi_{1}, \xi_{2}, \ldots, \xi_{m}\right)
$$

is a generator in $\mathscr{A}\left(\mathcal{W}_{m+1}\right)$ which does not belong to $\mathscr{A}\left(\mathcal{W}_{m}\right)$.

The product action of a Lie group $G$ on $\mathscr{E}$ prolongs in a natural manner to any multijet bundle $\mathscr{E}(\mathscr{E})$. Using the natural prolongation of one-parameter local diffeomorphisms on the target space to the jet bundles, we find the prolongation of fundamental vector fields of a group action. Let $X_{a}$ be a fundamental vector field on $V$; with respect to the coordinates we set

$$
X_{a}=\sum_{i} A_{a}^{i} \partial_{x^{i}}
$$

The corresponding fundamental vector field on $\mathscr{E}_{r}$ becomes

$$
X_{a}=\sum_{\alpha} \sum_{i} A_{a, \alpha}^{i} \partial_{\xi_{\alpha}^{i}}
$$

where $A_{a, \alpha}^{i}=A_{a}^{i}\left(x^{i}=\xi_{\alpha}^{i}\right)$. The prolongation of the fundamental vector field onto the multijet bundle $\mathscr{g}^{\left(k_{1}, \ldots, k_{r}\right)}\left(\mathscr{E}_{r}\right)=\prod_{\alpha} J^{k_{\alpha}} V_{\alpha}$ is obtained from (4.2). 


\section{The first-order transformations groups}

\subsection{The linear subgroups of $\mathrm{Gl}(n)$}

6.1.1. Invariants for $O(n)$. Let $\mathbf{e}=\left(e_{i}\right), i=1, \ldots, n$, be an orthonormal basis, $\left(\xi^{i}\right)$ the corresponding coordinates on $V$, and 2 the Euclidean quadratic form on $V$.

ZERO-ORDER INVARIANTS. Let $\hat{\xi}=\left(\xi_{1}, \xi_{2}, \ldots, \xi_{r}\right) \in \mathscr{E}_{r}$, with $\mathscr{E}_{r}=V_{1} \times V_{2} \times$ $\cdots \times V_{r}$. From Theorem 2.1, we know that each polynomial in the $r(r+1) / 2$ forms $2\left(\xi_{\alpha}, \xi_{\beta}\right)$ is invariant. Let $\mathcal{W}$ be the subset in $\mathscr{E}_{r}$ such that each $\xi \in \mathscr{W}$ is a linear independent set of points in $E^{n}$.

The following properties are elementary. We present them without proof.

Proposition 6.1. (1) The set $\mathscr{I}_{\mathscr{W}}=\left\{2\left(\xi_{\alpha}, \xi_{\beta}\right) \mid \alpha \leq \beta, \xi_{\alpha}, \xi_{\beta} \in \xi, \xi \in \mathscr{W}\right\}$ is functionally independent.

(2) The order of stabilization equals $r=n-1$. Moreover, if $r=n-1$, the polynomial invariants for $\mathrm{SO}(n)$ coincide with those for $O(n)$ as a consequence of Theorem 2.1.

(3) Let $\Gamma$ be a level set of $\Phi_{W}$ in $\mathscr{E}_{n-1}$. Then, $\Gamma$ is an orbit of $\mathrm{SO}(n)$.

It follows that the subset $\mathscr{W}$ in $\mathscr{E}_{n-1}$ is foliated by orbits of $\mathrm{SO}(n)$, and the leaves are the level surfaces of $\mathscr{I}_{W}$. Let $\mathscr{A}\left(\mathscr{W}_{W}\right)$ be the sheaf generated by $\mathscr{I}_{\mathscr{W}}$ in $\mathscr{E}_{n-1}$.

REMARK 6.2. Because $\mathscr{E}_{r}$ is the zero-order level in jet space, a curve $\gamma(t)$ in $\mathscr{W}$ is lying in an orbit if and only if the invariant sheaf $\mathscr{A}(\mathcal{W})$ restricted to $\gamma$ is constant.

Let $\xi=\left\{\xi_{1}, \ldots, \xi_{n-1}\right\}$ be a set of $n-1$ points in $V$. The isotropy group at $\xi_{n-1}$ is the group $\mathrm{SO}(n-1)$, and, by recursion, the isotropy group leaving $k$ points fixed, say $\left\{\xi_{n-k}, \xi_{n-k+1}, \ldots, \xi_{n-1}\right\}$, is the group $\mathrm{SO}(n-k)$ [2]. From this, it follows that, if $\gamma(t)$ is a nowhere constant layer curve such that the invariant sheaf restricted to $\gamma(t)$ is constant, the image of $\gamma(t)$ is locally the image of an orbit of $\mathrm{SO}(2)$.

Finally, remark that, if the symmetry of the $r$ points is taken into account, it is indicated to construct the quotient space $\mathcal{W} / \mathscr{S}$, where $\mathscr{S}$ is the symmetry group of $\mathscr{E}_{r}$. The set $\mathscr{W} / \mathscr{Y}$ is parameterized by

$$
0<2\left(\xi_{1}, \xi_{1}\right) \leq 2\left(\xi_{2}, \xi_{2}\right) \leq \cdots \leq 2\left(\xi_{r}, \xi_{r}\right)
$$

FIRST-ORDER INVARIANTS. In order to construct first-order invariants, we consider multijet space

$$
\mathscr{g}^{(1, \ldots, 1)}\left(\mathscr{E}_{r}\right)=J^{1} V_{1} \times J^{1} V_{2} \times \cdots \times J^{1} V_{r},
$$


which is a bundle over $\mathscr{E}_{r}$ with map $\pi: \mathscr{f}^{(1, \ldots, 1)}\left(\mathscr{E}_{r}\right) \rightarrow \mathscr{E}_{r}$. We denote by $\left(\left(\xi_{\alpha}, \dot{\xi}_{\alpha}\right)\right)$ the coordinates on $\mathscr{g}^{(1, \ldots, 1)}\left(\mathscr{E}_{r}\right)$. The action of $\mathrm{SO}(n)$ extends in a natural way to $\mathscr{g}^{(1, \ldots, 1)}\left(\mathscr{E}_{\gamma}\right)$ as $\left(g,\left(\xi_{\alpha}, \dot{\xi}_{\alpha}\right)\right) \mapsto\left(g \cdot \xi_{\alpha}, g \cdot \dot{\xi}_{\alpha}\right)$. Let $\mathcal{W}_{(1,1, \ldots, 1)}$ be the regular subset of $\mathscr{g}^{(1, \ldots, 1)}\left(\mathscr{E}_{r}\right)$.

The total derivative operators in the different layers $T_{\alpha}, \alpha=1, \ldots, r$ commute with the product action of $\mathrm{SO}(n)$. Then, if $\phi$ is an invariant function on $\mathscr{E}_{r}$, each function $T_{\alpha} \phi$ is invariant. $T_{\alpha} \phi$ is the $\alpha$-layer first-order prolongation of $\phi$. Let $\mathcal{W}^{\prime}=\mathcal{W}_{(1, \ldots, 1)} \cap \pi^{-1} \mathcal{W}$. We construct the invariant sheaf $\mathscr{A}^{(1)}\left({ }^{\mathcal{O}} \mathcal{W}^{\prime}\right)$ as the sheaf generated by the elements of

$$
\mathscr{I}_{\mathscr{W}}^{(1)}=\left\{2\left(\xi_{\alpha}, \xi_{\beta}\right), T_{\gamma} \mathscr{2}\left(\xi_{\alpha}, \xi_{\beta}\right) \mid \alpha \leq \beta, \xi_{\alpha}, \xi_{\beta} \in \xi, \xi \in W\right\},
$$

where $\alpha, \beta$, and $\gamma=1, \ldots, r$, restricted to $\mathcal{W}^{\prime}$.

We remark that $\mathscr{A}^{(1)}\left(\mathcal{W}^{\prime}\right)$ is generated by the $n(n-1) / 2$ forms $2\left(\xi_{\alpha}, \xi_{\beta}\right)$ and the $(n-1)(n-1)$ forms $2\left(\dot{\xi}_{\alpha}, \xi_{\beta}\right)$. The forms $2\left(\dot{\xi}_{\alpha}, \xi_{\beta}\right)$ are linear in the variables $\left(\dot{\xi}_{\alpha}\right)$, and because the points $\xi_{\beta}$ are linear independent, we find the following proposition.

Proposition 6.3. The rank of the sheaf $\mathbb{A}^{(1)}\left({ }^{\prime}{ }^{\prime}\right)$ equals $(n-1)(3 n-2) / 2$.

Remark that the dimension of the orbits equals $n(n-1) / 2$ and is constant on $\mathcal{W}$, while $\mathcal{W}$ has dimension $n(n-1)$.

Let $\mathcal{T}$ be a level surface of the invariant sheaf $\mathscr{A}^{(1)}\left({ } W^{\prime}\right)$. Then, $\mathscr{T}$ is foliated by orbits of $\mathrm{SO}(n)$. As a consequence of former Proposition 6.3, the codimension of the orbits in $\mathcal{T}$ equals $n-1$. This implies that we need $n-1$ extra independent generators to construct the complete invariant sheaf.

In order to derive $n-1$ extra invariants, we construct an invariant normal bundle $N(\mathcal{W})$, as subbundle of $T \mathcal{W}$. This bundle is a vector bundle whose sections are transversal to the leaves and which is invariant as a subbundle under the action of $\mathrm{SO}(n)$.

The fundamental vector fields on $\mathscr{E}_{n-1}$ for $\mathrm{SO}(n)$ are given by

$$
\left\{X_{a} ; a=1, \ldots, \frac{n(n-1)}{2}\right\}=\left\{X_{i j}=\sum_{\alpha=1}^{n-1} \xi_{\alpha}^{i} \partial_{\xi_{\alpha}^{j}}-\xi_{\alpha}^{j} \partial_{\xi_{\alpha}^{i}} ; i<j, i, j=1, \ldots, n\right\} .
$$

Proposition 6.4. The following set of $n(n-1) / 2$ vector fields commute with the fundamental vector fields $X_{a}$ on $\mathscr{E}_{r}$ :

$$
Y_{\alpha}=\sum_{i} \xi_{\alpha}^{i} \partial_{\xi_{\alpha}^{i}}, \quad Y_{\alpha, \beta}=\sum_{i} \xi_{\alpha}^{i} \partial_{\xi_{\beta}^{i}}-\xi_{\beta}^{i} \partial_{\xi_{\alpha}^{i}}
$$

Denote this set by $Y_{a}, a=1, \ldots, n(n-1) / 2$. A simple calculation, together with Lemma 3.1, shows the following proposition.

Proposition 6.5. The set of vector fields $Z_{a}=\left(X_{1}, X_{2}, \ldots, Y_{1}, Y_{2}, \ldots\right)$, with $a=1, \ldots, n(n-1)$, is linear independent at each point on $\mathcal{W}$. 
The normal bundle $N(\mathcal{W})$ is the subbundle of the tangent bundle spanned by the vector fields $Y_{a}$. The fibre of the bundle $N(\mathscr{W})$ is isomorphic to $\mathbb{R}^{n(n-1) / 2}$. Because the action of $\mathrm{SO}(n)$ is effective on $\mathcal{W}$, the group $\mathrm{SO}(n)$ acts trivially on $N(\mathcal{W})$. Let $L(\mathcal{W})$ be the subbundle of $T \mathcal{W}$ which is tangent to orbits and hence is spanned by the fundamental vector fields $X_{a}$. We define an invariant metric on $\mathcal{W}$ as

$$
\hat{q}_{2}\left(Y_{a}, Y_{b}\right)=\delta_{a b}, \quad \hat{q}_{2}\left(Y_{a}, X_{b}\right)=0, \quad \hat{q}_{2}\left(X_{a}, X_{b}\right)=K_{a b},
$$

where $K_{a b}$ is the Killing form on the Lie algebra $\mathfrak{s o}(n)$.

The lift of the semi-Riemannian tensor field $\hat{q}_{2}$ is a function $\tilde{q}_{2}$ on the tangent space $T \mathscr{W}$. Using the natural embedding

$$
J^{(1,1, \ldots, 1) \mathscr{E}_{n-1}} \stackrel{\stackrel{\imath}{\hookrightarrow}}{\rightarrow} \mathscr{\mathscr { E }}_{n-1},
$$

the function $\imath^{*} \tilde{q}_{2}$ is an invariant function on $\mathcal{W}^{\prime}$.

From the invariant metric, we construct invariant layer metrics. Let $\hat{q}_{2}^{\alpha}$ be the restriction of the metric $\hat{q}_{2}$ to the fibre of the $\alpha$ layer

$$
V_{1} \times \cdots \times T V_{\alpha} \times \cdots \times V_{r} \longrightarrow \mathscr{E}_{r}
$$

The function $\tilde{q}_{2}^{\alpha}=\imath^{*} \hat{q}_{2}^{\alpha}$ will, by abuse of language, be called the $\alpha$-layer

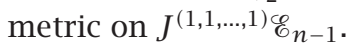

Because the subset $\mathscr{W}^{\prime}$ is dense in $\mathcal{W}_{(1, \ldots, 1)}$, the sheaf $\mathscr{A}^{(1)}\left(\mathcal{W}^{\prime}\right)$ extends in a unique way on $\mathcal{W}_{1, \ldots, 1}$, which determines the invariant sheaf.

THEOREM 6.6. The sheaf of invariant functions $\mathscr{A}\left(\mathcal{W}_{(1, \ldots, 1)}\right)$ for the action of $\mathrm{SO}(n)$ is generated by the extension of $\mathcal{A}^{(1)}\left({ }^{\mathfrak{W}} W^{\prime}\right)$ on $\mathcal{W}_{(1, \ldots, 1)}$ together with the $n-1$ layer metrics $\tilde{q}_{2}^{\alpha}$.

Proof. Because differentials of the layer metrics with respect to the coordinates $\dot{\xi}_{\alpha}$ are linear in the fibre coordinates $\dot{\xi}_{\alpha}$, we find functional independence of the layer metrics with respect to the sheaf generators of $\mathscr{A}^{(1)}\left({ }^{\prime}{ }^{\prime}\right)$. Moreover, they are functional independent among each other which proves the proposition.

The above constructed generators of $\mathscr{A}\left(\mathscr{W}_{1, \ldots, 1}\right)$ is given by the set

$$
\mathscr{I}\left(\mathcal{W}_{(1, \ldots, 1)}\right)=\left\{2\left(\xi_{\alpha}, \xi_{\beta}\right) ; 2\left(\dot{\xi}_{\alpha}, \xi_{\beta}\right) ; \tilde{q}_{2}^{\alpha}\right\}
$$

and relies entirely on the existence of the Killing form on the semi simple Lie algebra of the orthogonal group. An alternative way to derive generators of the invariant sheaf is using Capelli's identities together with the fact that the action of the linear group extended to jet bundles is linear. A straightforward application of these results yields the following theorem. 
THEOREM 6.7. The invariant sheaf $\mathscr{A}\left({ }^{\circ} W_{(1, \ldots, 1)}\right)$ is generated by the following set of functionally independent functions:

$$
\mathscr{C}\left(\mathscr{W}_{(1, \ldots, 1)}\right)=\left\{2\left(\xi_{\alpha}, \xi_{\beta}\right) ; 2\left(\dot{\xi}_{\alpha}, \xi_{\beta}\right) ; 2\left(\dot{\xi}_{\alpha}, \dot{\xi}_{\alpha}\right)\right\}
$$

Remark that $J^{(1,1, \ldots, 1) \mathscr{E}_{n-1}}$ contains a lot of redundant information. A large part of the invariant sheaf $\mathscr{A}\left(\mathcal{W}_{(1, \ldots, 1)}\right)$ can be retrieved from a smaller part of the sheaf. The following proposition is a direct consequence of the prolonged group action of $\mathrm{SO}(n)$.

Proposition 6.8. The subspace $\mathscr{V}=\left\{\dot{\xi}_{\beta}=0, \beta=2,3, \ldots, n-1, \dot{\xi}_{1} \neq 0\right\}$ is an invariant subspace in $J^{(1,1, \ldots, 1) \mathscr{E}_{n-1}}$.

The extension of the invariant sheaf $\mathscr{A}\left(\mathcal{W}_{(1, \ldots, 1)}\right)$ on the subspace $\mathscr{V}$ is an invariant sheaf, say $\mathscr{B}(\mathscr{V})$. With the canonical embedding

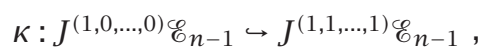

where $\kappa$ identifies $J^{(1,0, \ldots, 0) \mathscr{E}_{n-1}}$ with $\mathscr{V}$, the pullback $\kappa^{*} \mathscr{B}(\mathscr{V})$ is the invariant

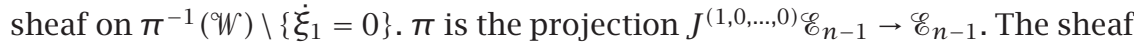
$\kappa^{*} \mathscr{B}(\mathscr{V})$ has a maximal rank.

Let $\triangle$ be the diagonal in $J^{(1,0, \ldots, 0) \mathscr{E}_{n-1}}$ defined by $\xi_{n-2}=\xi_{n-1}$ and

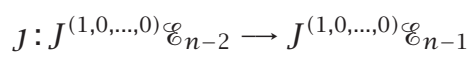

the diagonal embedding with image $\triangle$.

The invariant sheaf $\mathscr{A}\left(\mathcal{W}_{(1, \ldots, 0)}\right)$ then equals $J^{*} \kappa^{*} \mathscr{B}(\mathscr{V})$. The following theorem is an immediate consequence of our construction. Denote by $\mathscr{W}_{1}$ the regular subset $\mathcal{W}_{(1,0, \ldots, 0)}$.

THEOREM 6.9. The following sets are equivalent sets of generators of the invariant sheaf $\mathscr{A}\left({ }^{(} W_{1}\right)$ :

$$
\begin{aligned}
& \mathscr{I}\left(W_{1}\right)=\left\{2\left(\xi_{\alpha}, \xi_{\beta}\right) ; \mathscr{2}\left(\dot{\xi}_{1}, \xi_{\beta}\right) ; \tilde{q}_{2}^{1}\right\}, \\
& \mathscr{C}\left(W_{1}\right)=\left\{2\left(\xi_{\alpha}, \xi_{\beta}\right) ; 2\left(\dot{\xi}_{1}, \xi_{\beta}\right) ; 2\left(\dot{\xi}_{1}, \dot{\xi}_{1}\right)\right\},
\end{aligned}
$$

with $\alpha, \beta=1, \ldots, n-1$.

The above results generalize to the first-order jets over several layers. We formulate this in Theorem 6.10.

Let

$$
\mathscr{g}^{(1, \ldots, 1,0, \ldots, 0) \mathscr{E}_{n-l-1}}=\underbrace{J^{1} V_{1} \times \cdots \times J^{1} V_{l}}_{1 \text { time }} \times V_{l+1} \times \cdots \times V_{n-l-1},
$$

where $1 \leq l<n-1$ and $\mathscr{W}^{(l)}=\mathscr{W}_{(1, \ldots, 1,0, \ldots, 0)}$ is the regular subset. 
THEOREM 6.10. The following sets are equivalent sets of generators of the invariant sheaf:

$$
\begin{aligned}
& \mathscr{I}^{(l)}\left(\mathcal{W}^{(l)}\right)=\left\{2\left(\xi_{\alpha}, \xi_{\beta}\right) ; 2\left(\dot{\xi}_{\sigma}, \xi_{\beta}\right) ; \tilde{q}_{2}^{\sigma}\right\}, \\
& \mathscr{C}^{(l)}\left(\mathscr{W}^{(l)}\right)=\left\{2\left(\xi_{\alpha}, \xi_{\beta}\right) ; 2\left(\dot{\xi}_{\sigma}, \xi_{\beta}\right) ; 2\left(\dot{\xi}_{\sigma}, \dot{\xi}_{\sigma}\right)\right\},
\end{aligned}
$$

with $\alpha, \beta=1, \ldots, n-l-1$ and $\sigma=1, \ldots, l$.

REMARK 6.11. The expression $2\left(\dot{\xi}_{\alpha}, \dot{\xi}_{\alpha}\right)$ is a tensor field on the $\alpha$ layer for each $\alpha$ and determines the well-known Euclidean arc length parameter along each layer curve.

HIGHER-ORDER INVARIANTS. Before formulating the general case, we consider in detail the one-layer case and work by induction on the order. Let for $1 \leq l<n-1$

$$
\mathscr{g}^{(l, 0, \ldots, 0)} \mathscr{E}_{n-l-1}=J^{l} V_{1} \times V_{2} \times \cdots \times V_{n-l-1},
$$

and let $\mathcal{W}_{l}=\mathcal{W}_{(l, 0, \ldots, 0)}$ be the regular subset. The following theorem is a consequence of the identities of Capelli and the linear action of the prolonged action.

THEOREM 6.12. Let $k, r=1, \ldots, l, \alpha, \beta=1, \ldots, n-l-1$. The set

$$
\mathscr{C}_{l}\left(\mathscr{W}_{l}\right)=\left\{2\left(\xi_{\alpha}, \xi_{\beta}\right) ; 2\left(\xi_{1}^{(k)}, \xi_{1}^{(r)}\right) ; 2\left(\xi_{1}^{(k)}, \xi_{\beta}\right)\right\}
$$

generates the invariant sheaf on $\mathcal{W}_{l}$.

It is easily verified that the set is functionally independent and invariant. The cardinal number of the set equals $(n-l-1)(n-l) / 2+l(l+1) / 2+l(n-l-1)=$ $n(n-1) / 2$. The dimension of $\mathscr{g}^{(l, 0, \ldots, 0) \mathscr{E}_{n-l-1}}$ equals $n(n-1)$. Hence, we find on $\mathcal{W}_{l}$

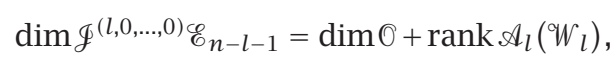

where $\mathscr{A}_{l}\left(\mathscr{W}_{l}\right)$ is the sheaf generated by $\Phi_{l}\left(\mathscr{W}_{l}\right)$.

Consider the space $\mathscr{g}^{(l+1,0, \ldots, 0) \mathscr{E}_{n-l-1}}=J^{l+1} V_{1} \times V_{2} \times \cdots \times V_{n-l-1}$ and projec-

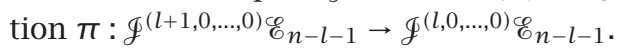

Let $\mathcal{W}^{\prime}=\mathcal{W}_{l+1} \cap \pi^{-1} \mathcal{W}_{l}$. The prolonged sheaf is generated by

$$
\mathscr{I}_{l}^{(1)}\left(\mathcal{W}^{\prime}\right)=\left\{2\left(\xi_{\alpha}, \xi_{\beta}\right) ; 2\left(\xi_{1}^{(k)}, \xi_{\beta}\right) ; 2\left(\xi_{1}^{(k)}, \xi_{1}^{(r)}\right)\right\}
$$

where $k=1, \ldots, l+1, r=1, \ldots, l, \alpha, \beta=1, \ldots, n-l-1$. 
The rank of $\mathscr{I}_{l}^{(1)}\left(\mathcal{W}^{\prime}\right)$ equals $n(n-1) / 2+(n-l-1)+l=n(n-1) / 2+n-1$. Using the unique extension of the sheaf on $\mathcal{W}_{l+1}$, we find that one generator is missing for the construction of the invariant sheaf on $\mathcal{W}_{l+1}$. For the construction of the extra generator, we follow the same procedure as in the first-order case. Because the group action of $\mathrm{SO}(n)$ extends linearly to the jet bundle, the same construction for the invariant transversal bundle can be applied at each order. From the invariant Killing metric on the orbits and an invariant metric in the normal bundle, we construct a layer metric on the first layer of multijet

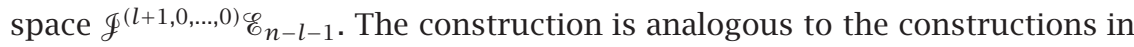
Propositions 6.4 and 6.5 .

Restriction of this metric to the tangent space to the jet bundle over the first layer gives us a layer metric. This metric lifts as a function on $\mathcal{W}_{l+1}$ denoted by $\tilde{q}_{2}^{1}$. We then have the following theorem.

THEOREM 6.13. The invariant sheaf $\mathscr{A}_{l+1}\left(\mathcal{W}_{l+1}\right)$ is generated by the set

$$
\mathscr{I}_{l}^{(1)}\left(\mathcal{W}_{l+1}\right),
$$

together with a layer metric $\tilde{q}_{2}^{1}$.

The construction is summarized in the following diagram:

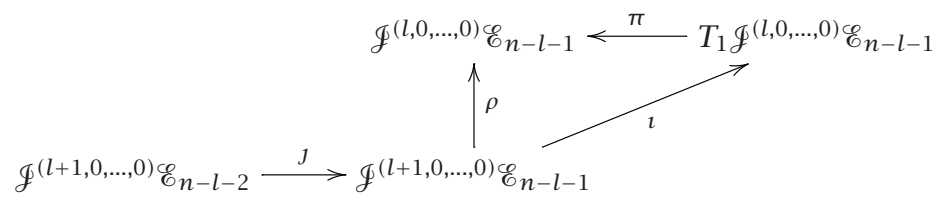

Here, $T_{1} \mathscr{F}^{(l, 0, \ldots, 0) \mathscr{E}_{n-l-1}}$ denotes the tangent bundle to the jet bundle over the first layer. $j$ is the diagonal embedding with image $\xi_{r-1}=\xi_{r}, \imath$ the natural embedding, and $\rho$ and $\pi$ the bundle projections.

COROLLARY 6.14. The following two sets are equivalent sets of generators:

$$
\begin{aligned}
& \mathscr{C}_{l+1}\left(\mathscr{W}_{l+1}\right)=\left\{2\left(\xi_{\alpha}, \xi_{\beta}\right) ; 2\left(\xi_{1}^{(k)}, \xi_{1}^{(r)}\right) ; 2\left(\xi_{1}^{(k)}, \xi_{\beta}\right)\right\}, \\
& \mathscr{I}_{l+1}\left(\mathscr{W}_{l+1}\right)=\left\{2\left(\xi_{\alpha}, \xi_{\beta}\right) ; 2\left(\xi_{1}^{(k)}, \xi_{\beta}\right) ; 2\left(\xi_{1}^{(k)}, \xi_{1}^{(r)}\right), \tilde{q}_{2}^{1}\right\},
\end{aligned}
$$

for $k, r=1, \ldots, l+1, \alpha, \beta=1, \ldots, n-l$.

TOP-ORDER INVARIANTS. The above construction works as long as $n-l-$ $1>1$. Then, $l=n-2$ determines what we call the top order. Proceeding as above, we have the following diagram:

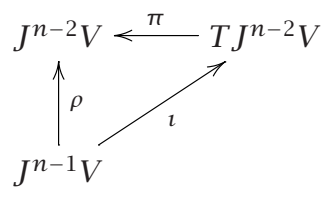


Notice that $\operatorname{dim} J^{n-2} V$ equals $n(n-1)$. Let $\mathscr{W}_{n-2}$ be the subset on which the fundamental vector fields are linear independent. The sheaf $\mathscr{A}_{n-2}\left(\mathscr{W}_{n-2}\right)$ is constructed the same way as before. Again, prolongation of the sheaf to $J^{n-1} V$ does not generate the invariant sheaf. An additional generator is constructed from the Killing form on the involutive distribution spanned by the fundamental vector fields, together with a metric in a normal invariant distribution spanned by a set of normal vector fields $Y_{a}$ as before. This determines an invariant metric $\hat{q}_{2}$ on $\mathcal{W}_{n-2}$. Let $\mathcal{W}_{n-1}$ be the open set in $J^{n-1} V$ on which the fundamental vector fields are linear independent. Let $\mathscr{A}(\mathcal{W})_{n-1}$ be the invariant sheaf generated in this way. The following theorem shows that we are really dealing with the top invariants.

THEOREM 6.15. Let $\mathscr{A}\left(W_{n-1}\right)$ be the invariant sheaf as defined above. Then, the invariant sheaf on $\mathcal{W}_{n+l-1}$ is generated by the lth prolongation of $\mathscr{A}\left({ }^{\mathrm{W}}\right)_{n-1}$ for $l>0$.

The theorem is a consequence of the dimensions of the jet spaces involved. Remark there is no restriction here because multispace contains only one layer.

6.1.2. Invariants for $\mathrm{CO}(n)$. The connected component of the identity of the group $\mathrm{CO}(n)$ is generated by $\mathrm{SO}(n)$, together with the dilatations $d_{\lambda}: \xi \mapsto$ $e^{\lambda} \xi$ with $\lambda \in \mathbb{R}$. Let $\mathscr{W} \subset \mathscr{E}_{n-1}$ be the subset of all sets of $n-1$ linear independent vectors in $V$, and $\mathscr{P}(\mathscr{W})$ the quotient space of $\mathscr{W}$ by the action of $d_{\lambda}$.

ZERO-ORDER INVARIANTS. Each invariant for $\mathrm{SO}(n)$ as formulated in Proposition 6.1, which is defined on $\mathscr{E}_{n-1}$, is a homogeneous monomial of degree two. For each $2\left(\xi_{\alpha}, \xi_{\beta}\right)$, we have $d_{\lambda}^{*} 2\left(\xi_{\alpha}, \xi_{\beta}\right)=e^{-2 \lambda} \mathscr{2}\left(\xi_{\alpha}, \xi_{\beta}\right)$. Consequently,

$$
\Phi_{\mathscr{W}}=\left\{2\left(\xi_{1}, \xi_{2}\right): 2\left(\xi_{1}, \xi_{3}\right): \cdots: 2\left(\xi_{n-1}, \xi_{n-1}\right)\right\}
$$

is a set of functionally independent functions on $\mathscr{P}(\mathcal{W})$ which are invariant under the action of $\mathrm{CO}(n)$.

HIGHER-ORDER INVARIANTS. The set of dilatations as subgroup of $\mathrm{Gl}(n)$ lifts linearly to any order in the jet bundle. The quotient space of the regular subset $\mathcal{W}_{(l, 0, \ldots, 0)}$ in $\mathscr{F}^{(l, 0, \ldots, 0)}\left(\mathscr{E}_{n-l-1}\right)$ by the action of $d_{\lambda}$ is the projectivization $\mathscr{P}\left(\mathcal{W}_{(l, 0, \ldots, 0)}\right)$ of $\mathcal{W}_{(l, 0, \ldots, 0)}$.

Hence, the projectivization of the set

$$
\Phi_{l}\left(W_{l}\right)=\left\{2\left(\xi_{\alpha}, \xi_{\beta}\right) ; 2\left(\xi_{1}^{(k)}, \xi_{1}^{(r)}\right) ; 2\left(\xi_{1}^{(k)}, \xi_{\beta}\right)\right\}
$$

is a set of generators of the invariant sheaf for $\mathrm{CO}(n)$. The same method determines generators for the top-order invariants. Their order equals $n-1$. We omit their derivation. 
REMARK 6.16. The invariant $2\left(\dot{\xi}_{1}, \dot{\xi}_{1}\right) / 2\left(\xi_{1}, \xi_{1}\right)$ is an invariant tensorfield on the first layer determining an invariant parameterization along first-layer curves.

REMARK 6.17. Dimension $n=2$ is exceptional. The sheaf of zero-order invariants is the constant sheaf. The group acts prehomogeneously on $V$, and the origin is the singular part. Because $J^{1} V \simeq V_{1} \times V_{2}$, a set of generators, is given by

$$
\mathscr{I}(\mathscr{W})=\{2(\xi, \xi): 2(\dot{\xi}, \xi): 2(\dot{\xi}, \dot{\xi})\},
$$

with $\mathcal{W}=J^{1} V \backslash\left\{\pi^{-1}(0)\right\}$, which generate the top-order invariants. Because the group $\mathrm{CO}(2)$ is Abelian, the geometric structure is locally flat.

6.1.3. Invariants for $\operatorname{Sp}(n)$. Let 2 be the skew-symmetric form on $V$ determining the symplectic structure, and e a basis of $V$. The dimension of $V$ is even and equals $2 n$. Recall that the dimension of the group equals $n(2 n+1)$.

ZERO-ORDER INVARIANTS. Let $\hat{\xi}=\left(\xi_{1}, \ldots, \xi_{r}\right) \in \mathscr{E}_{r}$. We know by Theorem 2.1 that the polynomials $2\left(\xi_{\alpha}, \xi_{\beta}\right)$, with $\alpha<\beta$, are invariant under the action of $\operatorname{Sp}(n)$. Let $\mathscr{W} \subset \mathscr{E}_{r}$ be the subset of all $r$ linear independent points in $V$. We then have the following lemma.

LEMmA 6.18. The set $\rrbracket_{\mathscr{W}}=\left\{2\left(\xi_{\alpha}, \xi_{\beta}\right) \mid \alpha<\beta, \xi_{\alpha}, \xi_{\beta} \in \xi, \xi \in W\right\}$ is functionally independent.

COROLLARY 6.19. The stabilization order for $\mathrm{Sp}(n)$ equals $r=2 n$.

The level surfaces of $\Phi_{\mathscr{W}}$ are submanifolds of constant dimension and, consequently, are disjoint unions of orbits. The generated sheaf is invariant and has rank $n(2 n-1)$. Each orbit is a pseudo-Riemannian manifold which inherits its geometric structure from the semi simple group $\operatorname{Sp}(n)$.

HIGHER-ORDER INVARIANTS. We show how to construct first-order invariants; then, higher-order invariants are derived by repeating the construction the way it has been done for the orthogonal group.

Consider the following jet bundle $\pi: \mathscr{f}^{(1,0, \ldots, 0)}\left(\mathscr{E}_{r}\right) \rightarrow \mathscr{E}_{r}$, with $r=2 n$, and construct the prolongation of $\mathscr{I}_{\mathscr{W}}$ on $\mathcal{W}^{\prime}=\pi^{-1}\left(\mathcal{W}^{\mathcal{W}}\right) \cap \mathcal{W}_{(1,0, \ldots, 0)}$, with $\mathcal{W}_{(1,0, \ldots, 0)}$ the regular subset of $\mathscr{g}^{(1,0, \ldots, 0)}\left(\mathscr{E}_{r}\right)$. Then,

$$
\mathscr{I}^{(1)}\left(\mathcal{W}^{\prime}\right)=\left\{2\left(\xi_{\alpha}, \xi_{\beta}\right), \alpha<\beta ; 2\left(\dot{\xi}_{1}, \xi_{\gamma}\right), \gamma \neq 1\right\} .
$$

Proposition 6.20. The set $\Phi^{(1)}\left({ }^{\prime} W^{\prime}\right)$ is functionally independent.

We extend the set $\mathscr{I}^{(1)}\left(\mathcal{W}^{\prime}\right)$ on $\mathcal{W}_{(1,0, \ldots, 0)}$. We easily find that, on $\mathcal{W}_{(1,0, \ldots, 0)}$, the subset of $\mathscr{f}^{(1,0, \ldots, 0)}\left(\mathscr{E}_{r}\right)$, we lack one generator. In order to construct an additional invariant, we need to construct an invariant normal bundle over the orbits in $\pi^{-1}\left({ }^{W}\right)$. 
The fundamental vector fields on $\mathcal{W}$ are given by

$$
\begin{aligned}
X_{j}^{i} & =\sum_{\alpha}\left[\xi_{\alpha}^{i} \partial_{\xi_{\alpha}^{j}}-\xi_{\alpha}^{n+j} \partial_{\xi_{\alpha}^{n+i}}\right], \\
X_{n+j}^{i} & =\sum_{\alpha} \frac{1}{2}\left[\xi_{\alpha}^{i} \partial_{\xi_{\alpha}^{n+j}}+\xi_{\alpha}^{j} \partial_{\xi_{\alpha}^{n+i}}\right], \\
X_{j}^{n+i} & =\sum_{\alpha} \frac{1}{2}\left[\xi_{\alpha}^{n+i} \partial_{\xi_{\alpha}^{j}}+\xi_{\alpha}^{n+j} \partial_{\xi_{\alpha}^{i}}\right] .
\end{aligned}
$$

Define the following vector fields on

$$
\begin{aligned}
\mathscr{W}: Y_{\beta}^{\alpha} & =\sum_{i}\left[\xi_{\alpha}^{i} \partial_{\xi_{\beta}^{i}}-\xi_{n+\beta}^{i} \partial_{\xi_{n+\alpha}^{i}}\right], \\
Y_{n+\beta}^{\alpha} & =\sum_{i} \frac{1}{2}\left[\xi_{n+\beta}^{i} \partial_{\xi_{\alpha}^{i}}-\xi_{\alpha}^{i} \partial_{\xi_{n+\beta}^{i}}\right], \\
Y_{\beta}^{n+\alpha} & =\sum_{i} \frac{1}{2}\left[\xi_{\beta}^{i} \partial_{\xi_{n+\alpha}^{i}}-\xi_{\alpha}^{i} \partial_{\xi_{n+\beta}^{i}}\right] .
\end{aligned}
$$

Proposition 6.21. The vector fields

$$
X_{j}^{i}, X_{j}^{n+i}, X_{n+j}^{i}, Y_{\beta}^{\alpha}, Y_{\beta}^{n+\alpha}, Y_{n+\beta}^{\alpha}
$$

are linear independent on $W$.

Let $q_{2}$ be the pseudo-Riemannian metric which, in the level sets, is given by the Killing form on the Lie algebra $\mathfrak{s p}(n)$ and, in the normal space, by an Euclidean metric. The restriction of this metric to the tangent space of the first layer defines the layer metric $q_{2}^{1}$. The lift of this metric, $\tilde{q}_{2}^{1}$, as a function on $\mathscr{g}^{(1,0, \ldots, 0)}(\mathcal{W})$, is an invariant function which is functionally independent from the set (6.27).

We are now able to formulate the following theorem on the regular subset $\mathcal{W}_{1}=W_{(1,0, \ldots, 0)}$.

THEOREM 6.22. The following sets are equivalent sets of generators of the invariant sheaf $\mathscr{A}\left(\mathcal{W}_{1}\right)$ :

$$
\begin{aligned}
& \mathscr{I}\left(\mathscr{W}_{1}\right)=\left\{2\left(\xi_{\alpha}, \xi_{\beta}\right), \alpha<\beta ; 2\left(\dot{\xi}_{1}, \xi_{\beta}\right), \beta \neq 1 ; \tilde{q}_{2}^{1}\right\}, \\
& \mathscr{C}\left(\mathscr{W}_{1}\right)=\left\{2\left(\xi_{\alpha}, \xi_{\beta}\right), \alpha<\beta ; 2\left(\dot{\xi}_{1}, \xi_{\beta}\right)\right\} .
\end{aligned}
$$

Consider the equivariant diagonal embedding defined by the diagonal $\xi_{2 n}=$ $\xi_{2 n-1}$

$$
J: \mathscr{f}^{(1,0, \ldots, 0)}\left(\mathscr{E}_{2 n-1}\right) \hookrightarrow \mathscr{J}^{(1,0, \ldots, 0)}\left(\mathscr{E}_{2 n}\right) .
$$

The pullback $\jmath^{*} \mathscr{I}\left(\mathcal{W}_{1}\right)$ determines a set of generators on the regular subset. Because the action of $\operatorname{Sp}(n)$ is linear, the same construction applies to all 
higher-order spaces $\mathscr{f}^{(l, 0, \ldots, 0)}\left(\mathscr{E}_{2(n-l)}\right)$. The top-order invariants are found for $l=n-1$.

REMARK 6.23. Each invariant $2\left(\dot{\xi}_{1}, \xi_{\beta}\right)$, for any $\beta$, determines an invariant parameters along first-layer curves. In case of the top order, the invariant $2\left(\dot{\xi}_{1}, \xi_{1}\right)$ is a good candidate.

\subsubsection{Invariants for $\operatorname{Sl}(n)$}

ZERO-ORDER INVARIANTS. Let $V$ be of dimension $n$, and consider the standard action of $\operatorname{Sl}(n)$ on $V$. The key invariant for $\operatorname{Sl}(n)$ is the determinant $\left|\xi_{1}, \ldots, \xi_{n}\right|$.

The following lemma shows that the order of stabilization equals $n$ in multispace $\mathscr{E}_{r}$.

LEMMA 6.24. Let $r<n$, and $\mathcal{O}$ an orbit of maximal dimension in $\mathscr{E}_{r}$. Then,

$$
\operatorname{dim} 0<n^{2}-1
$$

Proof. The proof follows from $\operatorname{dim}_{\mathscr{E}} \mathscr{E}_{r} \leq n(n-1)$ for $n>1$ and $r<n$.

The regular subset $\mathscr{W}$ of $\mathscr{E}_{n}$ is defined by the set of points $\xi=\left(\xi_{1}, \ldots, \xi_{n}\right)$ which is linear independent. Let $\mathscr{A}(\mathcal{W})$ be the invariant sheaf generated by $\mathscr{I}_{\mathscr{W}}=$ $\left\{\left|\xi_{1}, \ldots, \xi_{n}\right|\right\}$. All orbits in $\mathcal{W}$ are of maximal dimension. Let $\Gamma$ be a connected component of a level set of $\Phi_{\mathscr{W}}$, then $\Gamma$ is an orbit of $\operatorname{Sl}(n)$ and hence is a connected pseudo-Riemannian manifold.

FIRST-ORDER INVARIANTS. Let $\mathscr{W}_{(1,0, \ldots, 0)}$ be the regular subset in $\mathscr{f}^{1,0, \ldots, 0 \mathscr{C}_{n}}$.

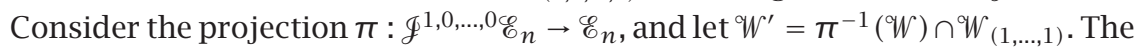
prolongation of $\mathscr{I}(\mathcal{W})$ to $\mathscr{W}^{\prime}$ is given by $\mathscr{I}^{(1)}\left(\mathcal{W}^{\prime}\right)=\left\{\left|\xi_{1}, \ldots, \xi_{n}\right|,\left|\dot{\xi}_{1}, \xi_{2}, \ldots, \xi_{n}\right|\right\}$.

Let $\mathscr{A}^{(1)}\left(\mathcal{W}^{\prime}\right)$ be the sheaf generated by $\mathscr{\Phi}^{(1)}\left({ }^{(} W^{\prime}\right)$. We easily find that

$$
\operatorname{dim} \mathscr{F}^{(1,0, \ldots, 0) \mathscr{E}_{n}}-\operatorname{dim} \operatorname{Sl}(n)-\operatorname{rk} \mathscr{A}^{(1)}\left(\mathscr{W}^{\prime}\right)=n-1,
$$

which implies that we are missing $n-1$ generators.

Supplementing the set of fundamental vector fields with the vector field $Y=\sum_{\alpha} \sum_{i} \xi_{\alpha}^{i} \partial_{\xi_{\alpha}^{i}}$ determines a complete parallelization of $\mathcal{W}$. Remark that the vector field $Y$ satisfies $\left[X_{a}, Y\right]=0$ and hence is invariant under the action of $\operatorname{Sl}(n)$.

Let $Z_{a}=\left\{X_{1}, X_{2}, \ldots, X_{n^{2}-1}, Y\right\}$, and set $Z_{a}=A_{a}^{\alpha i} \partial_{\xi_{\alpha}^{i}}$. We define the one forms $\omega^{a}=B_{\alpha i}^{a} d \xi_{\alpha}^{i}$, where $B=\left(B_{\alpha i}^{a}\right)$ is the inverse matrix of $A=\left(A_{a}^{\alpha i}\right)$. Using the chosen basis for the Lie algebra determines the canonical one form

$$
\omega: T^{\mathscr{W}} \longrightarrow \mathfrak{s}\{(n) \oplus \mathbb{R} .
$$

In particular, for any layer curve $\gamma(t)$ in $\mathcal{W}$ with running point in the first layer, we find $\omega(\dot{\gamma}(t)) \in \mathfrak{s}\left\{(n) \oplus \mathbb{R}\right.$. Remark that $\dot{\gamma}(t)$ is a point in $\mathcal{W}^{\prime}$ for fixed $t$. 
We determine $\left(\xi_{1}, \ldots, \xi_{n}, \dot{\xi}_{1}\right)$ as coordinates on $\mathscr{g}^{(1,0, \ldots, 0)}\left(\mathscr{E}_{n}\right)$. Let $q_{2}, q_{3}, \ldots, q_{n}$ be a base for the invariant polynomes on $\mathfrak{s}(n)$. The invariant polynomes on $\mathfrak{s}\left\{(n)\right.$ extend in a trivial manner to $\mathfrak{s}\left\{(n) \oplus \mathbb{R}\right.$. Let $\mathcal{W}_{1}=\mathscr{W}_{(1,0, \ldots, 0)}$. The functions

$$
\tilde{q}_{j}^{1}\left(\xi_{1}, \ldots, \xi_{n}, \dot{\xi}_{1}\right)=\left(\omega\left(\dot{\xi}_{1}\right)\right)^{*} q_{j}\left(\xi_{1}, \ldots, \xi_{n}\right)
$$

are functionally independent invariants on $\mathcal{W}_{1}$, for $j=2, \ldots, n$.

Proposition 6.25. The following sets are equivalent sets of generators of the invariant sheaf $\mathscr{A}\left(\mathcal{W}_{1}\right)$ :

$$
\begin{aligned}
\mathscr{I}\left(\mathscr{W}_{1}\right)= & \left\{\left|\xi_{1}, \ldots, \xi_{n}\right|,\left|\dot{\xi}_{1}, \ldots, \xi_{n}\right|, \tilde{q}_{2}^{1}, \ldots, \tilde{q}_{n}^{1}\right\} \\
\mathscr{C}\left(\mathscr{W}_{1}\right)= & \left\{\left|\xi_{1}, \xi_{2}, \ldots, \xi_{n}\right|,\left|\dot{\xi}_{1}, \xi_{1}, \xi_{3}, \ldots, \xi_{n}\right|,\right. \\
& \left.\left|\dot{\xi}_{1}, \xi_{2}, \xi_{1}, \ldots, \xi_{n}\right|, \ldots,\left|\dot{\xi}_{1}, \xi_{2}, \xi_{3}, \ldots, \xi_{1}\right|\right\} .
\end{aligned}
$$

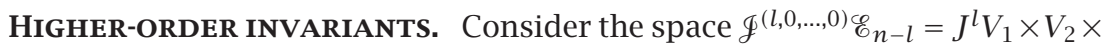
$\cdots \times V_{n-l}$. Using Capelli's results, we find a generating set on the regular subset

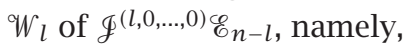

$$
\mathscr{C}\left(\mathscr{W}_{l}\right)=\left\{\left|\xi_{1}, \xi_{1}^{(1)}, \ldots, \xi_{1}^{(l)}, \xi_{2}, \ldots, \xi_{n-l}\right|\right\}
$$

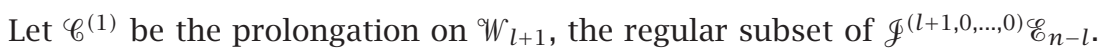
The dimension of $\mathscr{C}^{(1)}$ equals 2 . Then, dimensional arguments show that we lack $n-1$ generators to produce the invariant sheaf on the regular subset in

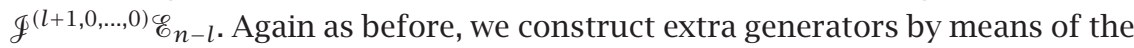
canonical one form on $\mathscr{g}^{(l, 0, \ldots, 0) \mathscr{E}_{n-l}}$ with values in $\mathfrak{s}\{(n) \oplus \mathbb{R}$ and a base for the invariant polynomials on $\mathfrak{s}(n)$. Restriction to the first layer considered as functions gives us the set $\left\{\tilde{q}_{2}^{1}, \tilde{q}_{3}^{1}, \ldots, \tilde{q}_{n}^{1}\right\}$.

Proposition 6.26. The following sets are equivalent sets of generators of the invariant sheaf $\mathscr{A}\left(\mathcal{W}_{l}^{(1)}\right)$ :

$$
\begin{aligned}
\mathscr{I}\left(\mathscr{W}_{l}^{(1)}\right)=\{ & \left|\xi_{1}, \xi_{1}^{(1)}, \ldots, \xi_{1}^{(l)}, \xi_{2}, \ldots, \xi_{n-l}\right|, \\
& \left.\left|\xi_{1}, \ldots, \xi_{1}^{(l-1)}, \xi^{(l+1)}, \xi_{2}, \ldots, \xi_{n-l}\right|, \tilde{q}_{2}^{1}, \ldots, \tilde{q}_{n}^{1}\right\}, \\
\mathscr{C}\left(\mathscr{W}_{l}^{(1)}\right)=\{\mid & \left|\xi_{1}, \xi_{1}^{(1)}, \ldots, \xi_{1}^{(l)}, \xi_{2}, \ldots, \xi_{n-l}\right|, \\
& \left|\xi_{1}, \xi_{1}^{(1)}, \ldots, \check{\xi}^{(k)}, \ldots, \xi^{(l+1)}, \xi_{2}, \ldots, \xi_{n-l}\right|, \ldots, \\
& \left.\left|\xi_{1}, \xi_{1}^{(1)}, \ldots, \xi_{1}^{(l+1)}, \xi_{2}, \ldots, \check{\xi}_{k}, \ldots, \xi_{n-l}\right|, \ldots\right\},
\end{aligned}
$$

where $\check{x}$ means the omission of the element $x$. 


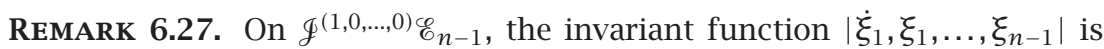
covariant of weight 1 under the action of the reparameterization group, while,

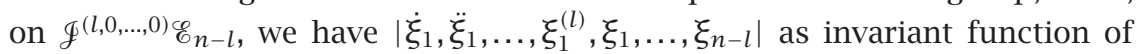
weight $l$ !.

THE TOP-ORDER INVARIANTS. Let $l=n-1$, and consider the space $J^{n-1} V$ equipped with the coordinates $\left(\xi, \xi^{(1)}, \ldots, \xi^{(n-1)}\right)$. The generator given by Capelli is the determinant $\Delta=\left|\xi, \xi^{(1)}, \ldots, \xi^{(n-1)}\right|$ on the regular subset $\mathscr{W}_{n-1}$, which is the subset where this determinant is nonzero. The level surfaces are the orbits of $\mathrm{SI}(n)$. Supplementing the set of fundamental vector fields with the vector field $Y=\sum_{k=0}^{n-1} \xi^{(k) i} \partial_{\xi^{(k) i}}$ determines a complete parallelisation of ${ }^{\mathcal{W}}{ }_{n-1}$. Let $\hat{\xi}=\left(\xi, \xi^{(1)}, \ldots, \xi^{(n)}\right)$ be a point in $\mathscr{W}_{n}$. The canonical one form $\omega$ takes values in $\mathfrak{s}\left[(n) \oplus \mathbb{R}\right.$ and pulls back the ad-invariant monomials $q_{i}, i=2, \ldots, n$, by

$$
\tilde{q}_{i}(\hat{\xi})=\omega\left(\dot{\xi}, \dot{\xi}^{(1)}, \ldots, \dot{\xi}^{n-1}\right)\left(\xi, \xi^{(1)}, \ldots, \xi^{n-1}\right)
$$

THEOREM 6.28. The following sets of invariant generators are equivalent:

$$
\begin{gathered}
\mathscr{S}\left(\mathscr{W}_{n}\right)=\left\{\Delta, \dot{\Delta}, \tilde{q}_{2}, \ldots, \tilde{q}_{n}\right\}, \\
\mathscr{C}\left(\mathcal{W}_{n}\right)=\left\{\Delta, \dot{\Delta},\left|\xi, \ldots, \check{\xi}^{(k)}, \ldots, \xi^{(n)}\right| ; k=0, \ldots, n\right\},
\end{gathered}
$$

where $\check{x}$ means the omission of the element $x$.

REMARK 6.29. The invariant function $\left|\xi_{1}, \dot{\xi}_{1}, \ldots, \xi_{1}^{(n-1)}\right|$ is of weight $(n-1)$ ! while $\left|\dot{\xi}_{1}, \ldots, \xi_{1}^{(n)}\right|$ is of weight $n$ ! under the action of reparameterizations.

6.1.5. Invariants for $\mathrm{Gl}(n)$. The connected component of the identity of the general linear group is generated by $\operatorname{Sl}(n)$, together with the dilatations $d_{\lambda}: \xi \mapsto e^{\lambda} \xi$ with $\lambda \in \mathbb{R}$. Let $\mathscr{W} \subset \mathscr{E}_{n}$ be the subset of all sets of $n$ linear independent vectors in $V$. The general linear group acts prehomogeneously on $\mathscr{W}$. Because the open orbit has maximal, the stabilization order of $\mathrm{Gl}(n)$ equals $n$. Hence, the zero-order invariant sheaf is constant.

HIGHER-ORDER INVARIANTS. The set of dilatations as subgroup of $\mathrm{Gl}(n)$ lifts linearly to any order in the jet bundle. From this, we find that the quotient space of the regular subset

$$
\mathcal{W}_{(l+1,0, \ldots, 0)} \subset \mathscr{F}^{(l+1,0, \ldots, 0)}\left(\mathscr{E}_{n-l}\right)
$$

by the action of $d_{\lambda}$ is the projectivization $\mathscr{P}\left(\mathcal{W}_{(l+1,0, \ldots, 0)}\right)$ of $\mathcal{W}_{(l+1,0, \ldots, 0)}$. The projectivization of the sets formulated in Proposition 6.26 gives two equivalent sets of generators of the invariant sheaf for $\operatorname{Gl}(n)$. The same method determines generators for the top-order invariants. Their order equals $n$. We omit their derivation. 
REMARK 6.30. The function

$$
\frac{\left|\dot{\xi}_{1}, \ldots, \xi_{1}^{(l+1)}, \xi_{2}, \ldots, \xi_{n-l}\right|}{\left|\xi_{1}, \dot{\xi}_{1}, \ldots, \xi_{1}^{(l)}, \xi_{1}, \ldots, \xi_{n-l}\right|},
$$

which is of weight $l+1$, determines an invariant parameter on $\mathcal{W}_{(l+1,0, \ldots, 0)}$.

6.2. The transitive transformation groups. We start with the Euclidean case and then indicate how the same constructions are applied to the other transitive groups. The central key in the construction is the introduction of an extra layer in a multispace and the homogenization of the invariants with respect to the zero-order coordinates in order to make them invariant under translations.

6.2.1. Invariants for the Euclidean transformation group. The orientation preserving Euclidean group is generated by $\mathrm{SO}(n)$, together with the translations. Let $\operatorname{dim} V=n$, and consider sets $\hat{\xi}=\left\{\xi_{1}, \ldots, \xi_{n}\right\}$ of $n$ points in $V$ such that the sets $\mathscr{Y}=\left\{\xi_{1}-\xi_{n}, \xi_{2}-\xi_{n}, \ldots, \xi_{1}-\xi_{n}\right\}$ are linear independent. Let $\eta_{\alpha}=\xi_{\alpha}-\xi_{n}$ for $\alpha=1, \ldots, n-1$. We define the following multispaces:

$$
\mathcal{N}_{n-1}=\prod_{\alpha=1}^{n-1} V_{\alpha}, \quad \mathscr{E}_{n}=\prod_{\alpha=1}^{n} V_{\alpha} .
$$

The space $\mathscr{E}_{n}$ is equipped with the coordinates $\hat{\xi}=\left(\xi_{\alpha}^{i}\right)$, and $\mathcal{N}_{n-1}$ with $\hat{\eta}=\left(\eta_{\alpha}^{i}\right)$. We define the projection $\rho: \mathscr{E}_{n} \rightarrow \mathcal{N}_{n-1}$ given by $\eta_{\alpha}=\xi_{\alpha}-\xi_{n}$ for $\alpha=1, \ldots, n-1$. Let $\mathscr{W}$ be the regular subset in $\mathcal{N}$; we define $\mathscr{V}=\rho^{-1} \mathscr{W}$ as subset of $\mathscr{E}_{n}$.

Proposition 6.31. The set of generating vector fields of the Euclidean group on $\mathscr{E}_{n}$ is linear independent at each point of $\mathscr{V}$.

Proof. Let $X_{a}$ be the generating vector fields on $\mathscr{E}$. The matrix of the components in terms of the coordinates is $\left(A_{a \alpha}^{i}\right)$, which takes the form

$$
\left(\begin{array}{llll}
\text { Id } & \text { Id } & \cdots & \text { Id } \\
A_{1} & A_{2} & \cdots & A_{n}
\end{array}\right)
$$

where each $A_{\alpha}$ is the matrix of the fundamental rotation vector fields in the layer $V_{\alpha}$. The proof follows from a rearrangement of the matrix such that

$$
\left(\begin{array}{cccc}
0 & 0 & \cdots & \text { Id } \\
A_{1}-A_{n} & A_{2}-A_{n} & \cdots & A_{n}
\end{array}\right) .
$$

ZERO-ORDER INVARIANTS. Using the results for the orthogonal group on $\mathcal{N}$, we find that the set $\Phi_{\mathscr{V}}=\rho^{*} \Phi_{\mathscr{W}}$ is functionally independent. $\mathscr{I}_{\mathscr{W}}$ is the set given by Proposition 6.1. Let $\mathscr{A}(\mathscr{V})$ be the sheaf generated by $\mathscr{I}_{\mathscr{W}}$. Because the level surfaces of $\mathscr{I}_{\mathscr{W}}$ are orbits of $\mathrm{SO}(n)$, the inverse image of an orbit of $\mathrm{SO}(n)$ 
in $\mathscr{W}$ by $\rho$ is an orbit of $\operatorname{Eucl}(n)$ and a level surface of $\mathscr{I}_{q}$. Hence, the regular subset $\mathscr{V}$ is foliated by orbits of $\operatorname{Eucl}(n)$.

Higher-ORDER INVARIANTS. Consider the first order and let $n \geq 2$. We construct the projection $\tilde{\rho}: \mathscr{F}^{(1,0, \ldots, 0)}\left(\mathscr{E}_{n}\right) \rightarrow \mathscr{g}^{(1,0, \ldots, 0)}\left(\mathcal{N}_{n-1}\right)$, which is given by $\eta_{\alpha}=\xi_{\alpha}-\xi_{n}, \dot{\eta}_{1}=\dot{\xi}_{1}$. Using the notations of Theorem 6.10 and defining $\mathscr{V}_{1}=$ $\tilde{\rho}^{-1} \mathcal{W}_{1}$, we find that the sets $\tilde{\rho}^{*} \mathscr{I}\left(\mathcal{W}_{1}\right)$ and $\tilde{\rho}^{*} \mathscr{C}\left(\mathcal{W}_{1}\right)$ are equivalent generators of the invariant sheaf $\mathscr{A}\left(\mathscr{V}_{1}\right)$.

The same construction extends to all orders, with $l \leq n-2$, using the projections $\tilde{\rho}: \mathscr{F}^{(l, 0, \ldots, 0)}\left(\mathscr{E}_{n-l}\right) \rightarrow \mathscr{\mathscr { F }}^{(l, 0, \ldots, 0)}\left(\mathcal{N}_{n-l-1}\right)$, which are given by $\eta_{\alpha}=\xi_{\alpha}-\xi_{n}$, $\eta_{1}^{(l)}=\xi_{1}^{(l)}$. In each case, it suffices to construct the pullback of the invariant generators on the regular subsets for $\mathrm{SO}(n)$.

REMARK 6.32. The invariant quadratic forms $2\left(\dot{\xi}_{\alpha}, \dot{\xi}_{\alpha}\right)$ determine the Euclidean arc length parameter along layer curves at any order.

TOP-ORDER INVARIANTS. Consider next the case $l=n-1$. For the space $\mathscr{g}^{(l, 0)}\left(\mathscr{E}_{2}\right)$, we have the invariant sheaf $\mathscr{A}(\mathscr{V})$, with $\mathscr{V}$ the regular subset of the jet space. Construction of the first layer prolongation yields $\mathscr{A}^{(1)}\left(\mathscr{V}^{(1)}\right)$, with $\mathscr{V}^{(1)}$ the inverse image of $\mathscr{V}$. Consider next the diagonal embedding $J: J^{(n-1)} V \rightarrow$ $J^{(n-1)} V \times V$, which is equivariant. The pullback of the set of generators of $\mathscr{A}^{(1)}\left(\mathscr{V}^{(1)}\right)$ by $J$ generates the invariant sheaf on the regular subset in $J^{(n-1)} V$. We omit the construction.

6.2.2. Invariants for the similarity transformation group. The similarity group is generated by $\mathrm{CO}(n)$, together with the translations. We use the same methods as for the Euclidean group. At zero order, we consider sets $\hat{\xi}=$ $\left\{\xi_{1}, \ldots, \xi_{n}\right\}$ of $n$ points in $V$ such that the sets $\mathscr{S}=\left\{\xi_{1}-\xi_{n}, \xi_{2}-\xi_{n}, \ldots, \xi_{n-1}-\right.$ $\left.\xi_{n}\right\}$ are linear independent. Let $\eta_{\alpha}=\xi_{\alpha}-\xi_{n}$ for $\alpha=1, \ldots, n-1$. The projection $\rho$ of $\mathcal{N}_{n-1}=\prod_{\alpha=1}^{n-1} V_{\alpha}$ to $\mathscr{E}_{n}=\prod_{\alpha=1}^{n} V_{\alpha}$ pulls back the generators (6.24). For the higher-order invariants, the same procedure applied to the set (6.25) gives the desired results. We omit the details.

REMARK 6.33. The invariants $2\left(\dot{\xi}_{\alpha}, \dot{\xi}_{\alpha}\right) / 2\left(\xi_{\alpha}-\xi_{\beta}, \xi_{\alpha}-\xi_{\beta}\right)$, for $\beta \neq \alpha$, determine invariant parameters along layer curves except for the top order where only one layer is available.

6.2.3. Invariants for the symplectic transformation group. The homogenization construction in this case is identical with the one used for the Euclidean group. We omit their construction.

6.2.4. Invariants for the volume-preserving transformation group. The volume-preserving group is generated by $\mathrm{Sl}(n)$, together with the translations. Via the map $\rho: \mathscr{E}_{n+1} \rightarrow \mathscr{E}_{n}$ as $\eta_{\alpha}=\xi_{\alpha}-\xi_{n+1}$, the pullback of the sets of invariants given in Proposition 6.25 determines a set of invariant generators on $\rho^{(-1)} \mathcal{W}$. For the higher-order invariants, we follow the same construction using the generators formulated in Proposition 6.26. To construct the top-order 
invariants, consider the jet bundle $J^{n} V \times V$ and the sheaf of invariants under the action of $\operatorname{SI}(n)$. Let $\mathscr{A}\left({ }^{\mathscr{W}}\right)$ be this sheaf. Construct the prolongation $\mathscr{A}^{(1)}\left(\mathcal{W}^{(1)}\right)$ with $\mathcal{W}^{(1)}=\pi^{(-1)} \mathcal{W}$. The pullback along the following maps yields the desired result: $J^{n+1} V \stackrel{J}{\rightarrow} J^{n+1} V \times V \stackrel{\kappa}{\rightarrow} J^{n+1} V$, where $J$ is the diagonal embedding and $\kappa$ the homogenization map $\eta=\xi_{1}-\xi_{2}$. Homogenization of the invariant parameters for the $\mathrm{Sl}(n)$ action yield invariant parameters. The same remains true at the top order.

6.2.5. Invariants for the affine transformation group. At the zero order, we take multispace $\mathscr{E}_{n+1}$ of rank $n+1$. Let $\rho: \mathscr{E}_{n+1} \rightarrow \mathscr{E}_{n}$ be the projection map defined by $\eta_{\alpha}=\xi_{\alpha}-\xi_{n+1}$, and consider the preimage of $\mathscr{W} \subset \mathscr{E}_{n}$. Because $\mathcal{W}$ is an orbit of $\operatorname{Gl}(n)$, we find that $\rho^{-1} \mathcal{W}$ is an orbit of the affine group. The invariant sheaf is consequently the constant sheaf.

Consider the projection $\rho: \mathscr{g}^{(l+1,0, \ldots, 0)}\left(\mathscr{E}_{n-l+1}\right) \rightarrow \mathscr{g}^{(l+1,0, \ldots, 0)}\left(\mathscr{E}_{n-l}\right)$. The invariants for $\operatorname{Gl}(n)$ are pulled back upon the former bundle via $\rho$. Then, homogenization with respect to the zero-order coordinates produces invariants with respect to the affine group. We omit their derivation. The same procedure applies to the top-order terms. The same remarks are valid for invariant parameters.

\section{The second-order transformation groups}

\subsection{The projective group}

7.1.1. Zero-order invariants. Recall that $\mathrm{Pl}(n)=\mathrm{Gl}(n+1) / \pm e^{\lambda} \cdot \mathrm{Id}_{n}$. Then, $\operatorname{dim} \operatorname{Pl}(n)=n(n+2)$. If $n$ is odd, we find $\operatorname{Pl}(n)=\operatorname{Sl}(n+1)$, while, in case $n$ is even, we find $\operatorname{Pl}(n)=\operatorname{Sl}(n+1) / \pm \operatorname{Id}$. By direct verification, we find that the stabilization order in $\mathscr{E}_{r}$ equals $n+2$.

PROPOSITION 7.1. Let $\Delta\left(k_{1}, \ldots, k_{n+1}\right)=\left|\xi_{k_{1}}-\xi_{k_{n+1}}, \ldots, \xi_{k_{n}}-\xi_{k_{n+1}}\right|$.

(a) The sets $\Delta\left(k_{1}, \ldots, k_{n+1}\right)=0$ are invariant sets.

(b) The singular subset $\Sigma \subset \mathscr{E}_{n+2}$ is defined by

$$
\prod_{k_{1}<k_{2}<\cdots<k_{n+1}} \Delta\left(k_{1}, \ldots, k_{n+1}\right)=0 .
$$

Proof. The set $\Delta\left(k_{1}, \ldots, k_{n+1}\right)=0$ is invariant under translations; hence, by means of a translation, we choose $\xi_{n+1}=0$. Using the transformation formula

$$
\tilde{\xi}^{i}=\frac{b_{j}^{i} \xi^{j}}{1+c_{i} \xi^{i}}=\frac{1}{f_{\xi}} b_{j}^{i} \xi^{j},
$$

we find

$$
\left|\tilde{\xi}_{k_{1}}, \ldots, \tilde{\xi}_{k_{n}}\right|=\frac{1}{f_{\xi_{k_{1}}}} \cdots \frac{1}{f_{\xi_{k_{n}}}}|b|^{n}\left|\xi_{k_{1}}, \xi_{k_{2}}, \ldots, \xi_{k_{n}}\right|,
$$


which proves statement (a). To prove (b), we remark that the set of fundamental vector fields drops rank at $\xi$ if and only if $\xi \in \Sigma$.

Proposition 7.2. The regular subset $\mathscr{W}=\mathscr{E}_{n+2} \backslash \Sigma$ is an orbit of $\operatorname{Pl}(n)$.

Proof. At each point of $W$, the set of fundamental vector fields span the tangent space. The singular subset $\Sigma$ is closed in $\mathscr{E}_{n+1}$; hence, $\mathscr{W}$ is an open subset. On the other hand, it is not hard to prove that, given two points $\hat{\xi}_{1}$ and $\hat{\xi}_{2}$ in $\mathcal{W}$, there exists an element in $g \in \operatorname{Pl}(n)$ such that $\hat{\xi}_{2}=g \cdot \hat{\xi}_{1}$. It suffices to show that the point $\left(\delta_{1}^{i}, \delta_{2}^{i}, \ldots, \delta_{n}^{i}, \sum_{k=1}^{n} \delta_{k}^{i}, 0\right)$ can be transformed into any $\left(\xi_{1}, \ldots, \xi_{n}, \xi_{n+1}, 0\right)$. Using the transformation (7.2), we find that

$$
\xi_{n+1}^{i}=\frac{\sum_{k=1}^{n} a_{k}^{i}}{1+\sum_{k=1}^{n} c_{k}}, \quad \xi_{k}^{i}=\frac{a_{k}^{i}}{1+c_{k}}
$$

for $k=1, \ldots, n$.

Rewriting this system as

$$
a_{k}^{i}=\xi_{k}^{i}\left(1+c_{k}\right), \quad \sum_{k=1}^{n}\left(\xi_{k}^{i}-\xi_{n+1}^{i}\right) c_{k}=\xi^{i}-\sum_{k=1}^{n} \xi_{k}^{i},
$$

we find that the determinant of this system is different from zero on $\mathcal{W}$, and hence, the system yields an unique solution.

COROLLARY 7.3. The sheaf of invariants on the zero-order level $\mathscr{W} \subset \mathscr{E}_{n+2}$ is constant.

Because the subset $\mathcal{W}$ is an orbit of the projective group, it inherits all the properties of the group. The set of fundamental vector fields $\left\{X_{a}\right\}$ has rank $n(n+2)$ on $\mathcal{W}$. Again, we define $X_{a}=A_{a}^{\alpha i} \partial_{\xi_{\alpha}^{i}}$. Let $B=\left(B_{\alpha i}^{a}\right)$ be the inverse matrix of $A=\left(A_{a}^{\alpha i}\right)$. The one form

$$
\omega=B_{\alpha i}^{a} d \xi_{\alpha}^{i} \otimes e_{a},
$$

where $\left(e_{a}\right)$ is a basis for the Lie algebra $\mathfrak{p} \mathfrak{\complement}(n)$, determines an isomorphism $\omega: T_{p} \mathcal{W} \rightarrow \mathfrak{p} \uparrow(n)$ for each $p \in \mathcal{W}$.

As a consequence, the $n$ canonical ad-invariant forms $\left\{q_{2}, \ldots q_{n+1}\right\}$ on $p \mathfrak{q}(n)$ define the invariant symmetric tensors

$$
\Phi_{i}=\operatorname{Tr}\left[(\operatorname{ad} \omega)^{i}\right],
$$

for $i=2, \ldots, n+1$. The form $\Phi_{2}$ defines a pseudo-Riemannian metric on $\mathcal{W}$.

7.1.2. Higher-order invariants. Consider the space $\mathscr{f}^{(1,0, \ldots, 0) \mathscr{E}_{n+2}}=J^{1} V_{1} \times$ $V_{2} \times \cdots \times V_{n+2}$ and the canonical identification $j: \mathscr{f}^{(1,0, \ldots, 0) \mathscr{E}_{n+2}} \rightarrow T V_{1} \times V_{2} \times$ $\cdots \times V_{n+2}$. Let $\Phi_{i}^{1}$ be the restrictions of the tensors $\Phi_{i}$ to the fibre of $\pi: T V_{1} \times$ $V_{2} \times \cdots \times V_{n+2} \rightarrow V_{1} \times V_{2} \times \cdots \times V_{n+2}$. The functions $j^{*} \Phi_{i}^{1}$ are invariant generators. The subset $\mathcal{W}_{(1,0, \ldots, 0)}=\pi^{-1} \mathcal{W} \backslash \Sigma_{1}$, where $\Sigma_{1}=\bigcup\left\{\left|\dot{\xi}_{1}, \xi_{k_{1}}, \xi_{k_{2}}, \ldots, \xi_{k_{n}}\right|=0\right\}$,

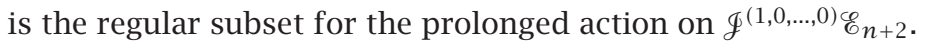


THEOREM 7.4. (1) The set $\mathscr{I}\left(\mathcal{W}_{(1,0, \ldots, 0)}\right)=\left\{j^{*} \Phi_{i}^{1}\right\}$ generates the invariant sheaf on $W_{(1,0, \ldots, 0)}$.

(2) The regular subset $W_{(1,0, \ldots, 0)}$ is foliated by orbits of $\mathrm{Pl}(n)$.

The construction applies to all higher orders.

LEMMA 7.5. The singular subset in $\mathscr{f}^{(l, 0, \ldots, 0) \mathscr{E}_{n-l+3}}$ is given by

$$
\Sigma_{l}=\bigcup_{1 \leq k_{1}<k_{2}<\cdots<k_{n-l+1}}\left\{\left|\xi_{1}^{(1)}, \ldots, \xi_{1}^{(l)} \xi_{k_{1}}, \ldots, \xi_{k_{n-l+1}}\right|=0\right\}
$$

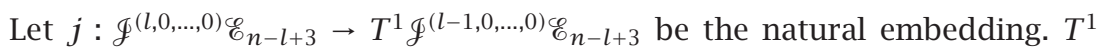
means the tangent bundle over the first layer. The set of ad-invariant symmetric tensors lift as functions to $T^{1} \mathscr{g}^{(l-1,0, \ldots, 0) \mathscr{E}_{n-l+3}}$. Let $\hat{\Phi}_{i}, i=2, \ldots, n+1$ be these functions. We then have the following theorem.

THEOREM 7.6. Let $\mathcal{W}_{(l, 0, \ldots, 0)}$ be the regular subset of $\mathscr{f}^{(l, 0, \ldots, 0) \mathscr{E}_{n-l+3}}$. The invariant sheaf on $\mathcal{W}_{(l, 0, \ldots, 0)}$ is generated by $\mathscr{I}\left(\mathcal{W}_{(l, 0, \ldots, 0)}\right)=\left\{j^{*} \hat{\Phi}_{i}, i=1, \ldots, n+1\right\}$.

The top-order invariants are obtained for $l=n+2$. Remark that, in this case, the tangent space to the first layer coincides with the tangent bundle of the jet bundle. The lifts of the ad-invariant forms are nothing else but their lifts to the tangent bundle of the group.

REMARK 7.7. In each case, the function $j^{*} \tilde{\Phi}_{2}$ determines a semi-Riemannian metric on the appropriate jet bundle over the first layer. Normalization of a layer curve with running point in the first layer fixes a projective invariant parameter on the curve. In case $n=1$, this parameter is determined by the Schwarzian derivative as will be shown in (7.14).

We present some results in low dimensions.

Projective Transformations ON $E^{1}$ [6]. Consider the action of $\mathrm{Sl}$ (2) on $E \equiv E^{1}=\mathbb{R}$. Let $\left(\partial_{x}, x \partial_{x}, x^{2} \partial_{x}\right)$ be fundamental fields on $E$.

(1) Consider $\mathscr{E}_{3}=E_{x} \times E_{y} \times E_{z}$. The fundamental vector fields on $\mathscr{E}_{3}$ are

$$
X_{1}=\partial_{x}+\partial_{y}+\partial_{z}, \quad X_{2}=x \partial_{x}+y \partial_{y}+z \partial_{z}, \quad X_{3}=x^{2} \partial_{x}+y^{2} \partial_{y}+z^{2} \partial_{z} .
$$

The singular subset is given by vanishing of the Vandermonden determinant $\Sigma:(y-x)(z-x)(z-y)=0$. Consider a regular layer curve $\hat{\gamma}(t)$ with running point $y(t)$ and lying in the regular subset $\mathscr{W}=\mathscr{E}_{3} \subset \Sigma$. Then, the norm of the tangent taken with respect to the layer metric $g$ is given by

$$
g\left(\hat{\gamma}^{\prime}, \hat{\gamma}^{\prime}\right)=2 \frac{(y-x)^{2}\left(y^{\prime}\right)^{2}}{(z-x)^{2}(z-y)^{2}},
$$

from which it follows that the curve is always lying outside the null cone of the metric. 
(2) Consider $\mathscr{E}\left(\mathscr{E}_{2}\right)=E_{x} \times J^{1} E_{y}$. The fundamental vector fields are

$$
X_{1}=\partial_{x}+\partial_{y}, \quad X_{2}=x \partial_{x}+y \partial_{y}+y^{\prime} \partial_{y^{\prime}}, \quad X_{3}=x^{2} \partial_{x}+y^{2} \partial_{y}+2 y y^{\prime} \partial_{y^{\prime}}
$$

The singular subset is given by $\Sigma=(y-x)^{2} y^{\prime}=0$. Again, we consider a regular layer curve $\hat{\gamma}(t)$ with running point $y(t)$ lying outside $\Sigma$. Then,

$$
g\left(\hat{\gamma}^{\prime}, \hat{\gamma}^{\prime}\right)=2\left[2 \frac{y^{\prime}}{y-x}-\frac{y^{\prime \prime}}{y^{\prime}}\right]^{2},
$$

with $g$ the layer metric. This curve is either lying outside the null cone or on the null cone of the metric.

(3) Finally, consider $\mathscr{g}\left(\mathscr{E}_{1}\right)=J^{3} E_{x}$. The fundamental vector fields are now

$$
\begin{aligned}
& X_{1}=\partial_{x}, \quad X_{2}=x \partial_{x}+x^{\prime} \partial_{x^{\prime}}+x^{\prime \prime} \partial_{x^{\prime \prime}}, \\
& X_{3}=x^{2} \partial_{x}+2 x x^{\prime} \partial_{x}+2\left(\left(x^{\prime}\right)^{2}+x x^{\prime \prime}\right) \partial_{x^{\prime \prime}},
\end{aligned}
$$

and singular subset $\Sigma:\left(x^{\prime}\right)^{3}=0$. Consider a regular curve $\gamma(t)$ in $E_{x}$. Then,

$$
g\left(\hat{\gamma}^{\prime}, \hat{\gamma}^{\prime}\right)=-4\left[\frac{x^{\prime \prime \prime}}{x^{\prime}}-\frac{3}{2}\left(\frac{x^{\prime \prime}}{x^{\prime}}\right)^{2}\right],
$$

which lies inside or outside the null cone of the metric. The last invariant equals minus 4 the well-known Schwarzian derivative in projective geometry [21, 26]. Notice that any regular curve in $E$ is in the regular subset of the projective action.

REMARK 7.8. In each of the cases, we have a parameter along the curve such that $g\left(\tilde{\gamma}^{\prime}, \tilde{\gamma}^{\prime}\right)=$ constant which determines a projective invariant parameter.

Projective Transformations on $E^{2}$. In this section, we study the projective motion of points in $E \equiv E^{2}=\mathbb{R}^{2}$. Let $x=\left(x^{1}, x^{2}\right)$ be the standard coordinates on $E$.

The one-parameter groups generated by the standard basis vectors in $\mathrm{Pl}(2)$ define through the action on $E$ the set of fundamental vector fields on $E$ given by

$$
\begin{gathered}
X_{1}=x^{2}\left(\sum_{i} x^{i} \partial_{i}\right), \quad X_{2}=-x^{1}\left(\sum_{i} x^{i} \partial_{i}\right), \quad X_{3}=x^{2} \partial_{1}, \\
X_{4}=-2 x^{1} \partial_{1}-x^{2} \partial_{2}, \quad X_{5}=-x^{1} \partial_{1}+x^{2} \partial_{2}, \\
X_{6}=\partial_{1}, \quad X_{7}=x^{1} \partial_{2}, \quad X_{8}=\partial_{2} .
\end{gathered}
$$


The invariant polynomials on the Lie algebra are given by the coefficients of the polynomial in $\lambda$

$$
\operatorname{det}\left[\left(\begin{array}{ccc}
d & b & a \\
f & -d+e & c \\
h & g & -e
\end{array}\right)-\lambda \operatorname{Id}_{3}\right]=0 .
$$

This yields the quadratic form

$$
q_{2}=d^{2}-d e+e^{2}+b f+a h+c g
$$

and the cubic form

$$
q_{3}=d e(d-e)+e(b f-a h)+d(a h-c g)+b c h+a f g .
$$

The Killing form equals 12 times $q_{2}$ as follows from a direct calculation.

The prehomogeneous spaces on which $\mathrm{Pl}(2)$ acts and which are used as reference spaces are (1) $\mathscr{E}_{4}=E_{x} \times E_{y} \times E_{u} \times E_{v}$, (2) $\mathscr{g}^{(1,0,0)}\left(\mathscr{E}_{3}\right)=J^{1} E_{x} \times E_{y} \times E_{u}$, (3) $\mathscr{g}^{(2,0)}\left(\mathscr{E}_{2}\right)=J^{2} E_{x} \times E_{y}$, (4) $\mathscr{g}^{(1,1)}\left(\mathscr{E}_{2}\right)=J^{1} E_{x} \times J^{1} E_{y}$, and $(5) \mathscr{g}^{3}\left(\mathscr{E}_{1}\right)=J^{3} E_{x}$.

From the quadratic and the cubic forms constructed for the prehomogeneous spaces, we are able to derive invariant generators on appropriate multijet spaces using the equivariant lifting procedure given in the chapter on jet bundles.

(1) $\mathscr{f}^{(1,0,0,0)}\left(\mathscr{E}_{4}\right)$. Taking the one jets of layer curves in $\mathscr{E}_{4}$ with running point in the $x$ layer, we find the space $\mathscr{g}^{(1,0,0,0)}\left(\mathscr{E}_{4}\right)$. Let $\pi: \mathscr{f}^{(1,0,0,0)}\left(\mathscr{E}_{4}\right) \rightarrow \mathscr{E}_{4}$ be the projection. The set $\Sigma:|x y u| \cdot|x y v| \cdot|x u v| \cdot|y u v|=0$ in $\mathscr{E}_{4}$ is the singular locus. Let $\left.q_{2}\right|_{x}$ and $\left.q_{3}\right|_{x}$ be the restrictions to the $x$ layer of the quadratic and cubic form on $\mathscr{E}_{4}$. These forms are not defined at $\Sigma$. Let $\mathscr{W}$ be the set in $\mathscr{f}^{(1,0,0,0)}\left(\mathscr{E}_{4}\right) \backslash \pi^{-1}(\Sigma)$. The lift of both forms are the desired generators.

(2) $\mathscr{g}^{(2,0,0)}\left(\mathscr{E}_{3}\right)$. Taking the one jets of layer curves in $\mathscr{g}^{(1,0,0)}\left(\mathscr{E}_{3}\right)$ with running point in the $x$ layer, we find the space $\mathscr{f}^{(2,0,0)}\left(\mathscr{E}_{3}\right)$. Let $\pi: \mathscr{f}^{(2,0,0)}\left(\mathscr{E}_{3}\right) \rightarrow$ $\mathscr{g}^{(1,0,0)}\left(\mathscr{E}_{3}\right)$ be the canonical projection. The singular subset $\Sigma$ in $\mathscr{g}^{(1,0,0)}\left(\mathscr{E}_{3}\right)$ is given by $|x y u|^{2}\left|x^{\prime}(u-x)\right|\left|x^{\prime}(y-x)\right|=0$. The quadratic and cubic forms restricted to the $x$ layer and lifted onto $\mathscr{W}=\mathscr{g}^{(2,0,0)}\left(\mathscr{E}_{3}\right) \backslash \pi^{-1}(\Sigma)$ generate the invariant sheaf on $\mathcal{W}$.

(3) $\mathscr{g}^{(1,1,0)}\left(\mathscr{E}_{3}\right)$. The set of one jets of layer curves in $\mathscr{g}^{(1,0,0)}\left(\mathscr{E}_{3}\right)$ with running point in the $y$ layer defines the space $\mathscr{g}^{(1,1,0)}\left(\mathscr{E}_{3}\right)$. Denote by $\pi: \mathscr{f}^{(1,1,0)}\left(\mathscr{E}_{3}\right) \rightarrow$ $\mathscr{g}^{(1,0,0)}\left(\mathscr{E}_{3}\right)$ the canonical projection. The quadratic and cubic forms defined outside the singular subset $\Sigma:|x y u|^{2}\left|x^{\prime}(u-x)\right|\left|x^{\prime}(y-x)\right|=0$ and restricted to the $y$ layer are invariant. Their lifts onto the set $\mathscr{W}=\mathscr{f}^{(1,1,0)}\left(\mathscr{E}_{3}\right) \backslash \pi^{-1}(\Sigma)$ define a set of generating functions of the invariant sheaf on $W$.

(4) $\mathscr{g}^{(3,0)}\left(\mathscr{E}_{2}\right)$. Consider the prehomogeneous space $\mathscr{g}^{(2,0)}\left(\mathscr{E}_{2}\right)$. The one jets of the layer curves with running point in the $x$ layer defines multijet space $\mathscr{g}^{(3,0)}\left(\mathscr{E}_{2}\right)$. The singular subset of this last space is given by $\Sigma:\left|x^{\prime} x^{\prime \prime}\right|^{3} \mid x^{\prime}(y-$ $x) \mid=0$. The quadratic and cubic forms on the regular part and restricted to 
the $x$ layer are invariant forms. Their lifts on $\mathcal{W}=\mathscr{g}^{(3,0)}\left(\mathscr{E}_{2}\right) \backslash \pi^{-1}(\Sigma)$, where $\pi$ is the projection $\mathscr{g}^{(3,0)}\left(\mathscr{E}_{2}\right) \rightarrow \mathscr{g}^{(2,0)}\left(\mathscr{E}_{2}\right)$ and generate the invariant sheaf on $\mathcal{W}$.

(5) $\mathscr{g}^{(2,1)}\left(\mathscr{E}_{2}\right)$. The set of one jets of layer curves with running point in the $x$ layer in the multijet space $\mathscr{g}^{(1,1)}\left(\mathscr{E}_{2}\right)$ defines the space $\mathscr{g}^{(2,1)}\left(\mathscr{E}_{2}\right)$. The singular subset $\mathscr{S}$ in $\mathscr{g}^{(1,1)}\left(\mathscr{E}_{2}\right)$ is given by $\Sigma:\left|x^{\prime}(y-x)\right|^{2}\left|y^{\prime}(x-y)\right|^{2}=0$. Define $\mathscr{W}=\mathscr{g}^{(2,1)}\left(\mathscr{E}_{2}\right) \backslash \pi^{-1}(\mathscr{S})$, with $\pi$ the projection $\mathscr{f}^{(2,1)}\left(\mathscr{E}_{2}\right) \rightarrow \mathscr{f}^{(1,1)}\left(\mathscr{E}_{2}\right)$. The quadratic and the cubic form restricted to the $x$ layer lifted onto $\mathcal{W}$ are generating functions of the invariant sheaf on $\mathcal{W}$.

(6) $\mathscr{f}^{4}\left(\mathscr{E}_{1}\right)$. Consider the one jets of curves in $\mathscr{g}^{3}\left(\mathscr{C}_{1}\right)$. The singular subset of $\mathscr{g}^{3}\left(\mathscr{E}_{1}\right)$ is given by $\Sigma:\left|x^{\prime} x^{\prime \prime}\right|^{4}=0$. Let $\pi$ be the projection $\mathscr{F}^{4}\left(\mathscr{E}_{1}\right) \rightarrow \mathscr{f}^{3}\left(\mathscr{E}_{1}\right)$. The quadratic and the cubic form lifted onto $\mathscr{W}=\mathscr{L}^{4}\left(\mathscr{E}_{1}\right) \backslash \pi^{-1}(\Sigma)$ determine the generating functions of the invariant sheaf over $\mathscr{W}$.

\subsection{The conformal group}

7.2.1. Zero-order invariants. We start with a classical result on the group of conformal transformations.

LEMMA 7.9 [1]. The group $\mathrm{CO}^{(1)}(n)$ is isomorphic to $O^{+}(1, n+1)$.

Here, $O^{+}(1, n+1)$ is the subgroup of $O(1, n+1)$ which preserves the null cone orientation in Minkowski space $E^{(1, n+1)} \cdot 2$ is the quadratic form with signature $(-,+, \ldots,+)$. We will identify the Lie algebra of the conformal group with the Lie algebra $o(1, n+1)$.

Using the isomorphism, we proceed as in the orthogonal case. Choose sets of $n+1$ points in $E^{1, n+1}$ and construct multispace $\mathscr{V}_{n+2}=\prod_{\alpha=1}^{n+2} E_{\alpha}^{(1, n+1)}$. Let $\left(z_{\alpha}^{i}\right): i=0, \ldots, n+1$ and $\alpha=1, \ldots, n+1$ be coordinates on this space. Let ${ }^{\prime} W^{\prime}$ be the regular subset in $\mathscr{V}$ consisting of linear independent sets of points in $E^{1, n+1}$. The future null cone at the origin $2\left(z_{\alpha}, z_{\alpha}\right)=0$ in each layer is invariant for the action of $O^{+}(1, n+1)$. Moreover, the group sends future-oriented null lines through the origin into future-oriented null lines through the origin in each layer space. We are interested in the set of invariants under the action of $O^{+}(1, n+1)$ on the $n+1$ product of $n$-spheres representing the future-oriented null lines. The null cones are given by $\left(z_{\alpha}^{0}\right)^{2}=\sum_{i}\left(z_{\alpha}^{i}\right)^{2}$. Let $\mathcal{N}^{+}=\prod_{\alpha=1}^{n+1} \mathcal{N}_{\alpha}^{+}$be the set of all null lines in this product. Choosing the polar point $\left(z_{\alpha}^{n+1}=1\right.$, $\left.z_{\alpha}^{i}=0\right), i \neq n+1$ in each layer, we find by stereographic projection the $(n+1)$ fold product of vector spaces $V_{\alpha}$. Multispace is given by $\mathscr{E}_{n+1}=\prod_{\alpha=1}^{n+1} V_{\alpha}$. Let $\xi_{\alpha}^{i}$ be the coordinates on $\mathscr{E}_{n+1}$ and 2 the Euclidean form on each layer. The embedding of $\mathscr{E}_{n+1}$ is given by $[1,4]$

$$
\sigma:\left(\xi_{\alpha}\right) \longmapsto\left(1, \frac{2 \xi_{\alpha}^{1}}{2\left(\xi_{\alpha}, \xi_{\alpha}\right)+1}, \frac{2 \xi_{\alpha}^{2}}{2\left(\xi_{\alpha}, \xi_{\alpha}\right)+1}, \ldots, \frac{2 \xi_{\alpha}^{n}}{2\left(\xi_{\alpha}, \xi_{\alpha}\right)+1}, \frac{2\left(\xi_{\alpha}, \xi_{\alpha}\right)-1}{2\left(\xi_{\alpha}, \xi_{\alpha}\right)+1}\right) .
$$

The image is the $(n+1)$-fold product of punctured $n$ dimensional spheres. We will denote the image also by $\sigma$. Let $P$ be the plane containing these spheres 
which is given by $z_{\alpha}^{0}=1, \alpha=1, \ldots, n+1$. A short calculation gives

$$
\sigma^{*} 2\left(z_{\alpha}, z_{\beta}\right)=-2 \frac{2\left(\xi_{\alpha}-\xi_{\beta}, \xi_{\alpha}-\xi_{\beta}\right)}{\left(2\left(\xi_{\alpha}, \xi_{\alpha}\right)+1\right)\left(2\left(\xi_{\beta}, \xi_{\beta}\right)+1\right)} .
$$

Hence, we find that all expressions of the form

$$
2\left(z_{\alpha}, z_{\beta}\right) 2\left(z_{\gamma}, z_{\delta}\right) / 2\left(z_{\alpha}, z_{\gamma}\right) 2\left(z_{\beta}, z_{\delta}\right)
$$

are invariants defined on $\mathcal{N}^{+}$. Set $q_{\alpha, \beta}=2\left(\xi_{\alpha}-\xi_{\beta}, \xi_{\alpha}-\xi_{\beta}\right)$. But, then, the functions $q_{\alpha, \beta} q_{\gamma, \delta} / q_{\alpha, \gamma} q_{\beta, \delta}$ are invariant on $\mathscr{E}_{n+1}$. Functionally independent sets are defined by recurrence. Let, for $\alpha<\beta$,

$$
\Phi_{\alpha, \beta, \delta}=\frac{q_{\alpha, \delta} \mathcal{q}_{\beta,(\beta+1)}}{\mathcal{q}_{\beta, \delta} q_{\alpha,(\beta+1)}}
$$

and define, for $\beta>3$,

$$
\begin{gathered}
\Phi_{\beta}=\left\{\Phi_{\alpha, \beta, \delta}, \Phi_{\alpha, \beta, \delta}^{-1} ; \alpha, \delta=(1,2),(2,3), \ldots,(\beta-2, \beta-1),(\beta-1,1)\right\}, \\
\mathscr{I}_{\mathscr{W}_{\beta+1}}=\mathscr{I}_{\mathscr{W}_{\beta}} \cup \Phi_{\beta},
\end{gathered}
$$

where $\mathcal{W}_{\beta}$ stands for the regular subset of the product of the $\beta$ first factors.

Let $r$ denote the number of factors in $\mathscr{E}_{r}$. We then have following results:

(1) $r=3$. The only invariants are constants;

(2) $r=4$. Let

$$
Q_{1}=\frac{q_{1,2} q_{3,4}}{q_{1,3} q_{2,4}}, \quad Q_{2}=\frac{q_{1,2} q_{3,4}}{q_{1,4} q_{2,3}} .
$$

The set $\Phi_{\mathscr{W}_{4}}=\left\{Q_{1}, Q_{2}, Q_{1}^{-1}, Q_{2}^{-1}\right\}$ is a set of invariant generators;

(3) $r=5$. Define

$$
Q_{3}=\frac{q_{1,2} q_{4,5}}{q_{4,2} q_{1,5}}, \quad Q_{4}=\frac{q_{2,3} q_{4,5}}{q_{4,3} q_{2,5}}, \quad Q_{5}=\frac{q_{3,1} q_{4,5}}{q_{4,1} q_{3,5}} .
$$

The set $\mathscr{I}_{\mathscr{W}_{5}}=\left\{Q_{i}, Q_{i}^{-1} ; i=1, \ldots, 5\right\}$ is a set of invariant generators.

Proposition 7.10. Provided $r \geq 3$, the number of generators in the set $\Phi_{W_{r}}$ equals $r(r-3) / 2$.

Proof. The number of functions in the set $\Phi_{\alpha}$ equals $\alpha-1$. Hence, the number of functions in $\mathscr{I}_{W_{n+1}}$ equals $\sum_{i=2}^{r-2} i$, which proves the proposition.

Using the results for the orthogonal group $O^{+}(1, n+1)$, we find that $\mathcal{W}_{n+1}$ is foliated by orbits of maximal dimension which are level surfaces of the invariant set $\mathscr{I}_{\mathscr{W}_{r}}$.

7.2.2. Higher-order invariants. In case $n=2$, the conformal group acts prehomogeneously on the space $\mathscr{E}_{3}$. Let $\mathscr{W}_{1}$ be the regular subset of $\mathscr{g}^{(1,0,0) \mathscr{E}_{3}}$, and 
$\omega$ the canonical one form on $\mathcal{W}$ with values in the Lie algebra $\mathfrak{c o}^{(1)}(2)$. Define

$$
\Phi_{1}=\operatorname{Tr}\left(\mathbf{a d}(\omega)^{2}\right), \quad \Phi_{2}=\operatorname{Tr}\left(\mathbf{a d}(\omega)^{4}\right) ;
$$

then, the set

$$
\Phi\left(W_{1}\right)=\left\{\tilde{\Phi}_{1}^{1}, \tilde{\Phi}_{2}^{1},\left(\tilde{\Phi}_{1}^{1}\right)^{(-1)},\left(\tilde{\Phi}_{2}^{1}\right)^{(-1)}\right\}
$$

where $\tilde{\Phi}_{1}^{1}, \tilde{\Phi}_{2}^{1}$ are the functions which are the lifts of the restriction of the symmetric tensors $\Phi_{1}$ and $\Phi_{2}$ to the tangent space to the first layer, is a set of invariant generators.

REMARK 7.11. The symmetric tensor $\Phi_{1}$ determines an invariant parameter along each curve in $\mathscr{W} \subset \mathscr{E}_{3}$.

Let $n>2$. In this case, the regular subset of multispace $\mathscr{E}_{n+1}$ is foliated by orbits of $\mathrm{CO}^{(1)}(n)$. In order to construct all invariants, we need the following construction.

Let $\mathcal{W}^{\prime}$ be the regular subset in multispace $\mathscr{V}=\prod_{\alpha=1}^{n+1} E^{(1, n+1)}$ and let $\left(z_{\alpha}^{i}\right)$, with $i=0,1, \ldots, n+1$ and $\alpha=1, \ldots, n+1$, be the standard coordinates. The following set of vector fields are fundamental fields for the action of $O^{+}(1, n+$ 1):

$$
X_{i j}=\sum_{\alpha=1}^{n+1}\left[z_{\alpha}^{i} \partial_{z_{\alpha}^{j}}-z_{\alpha}^{j} \partial_{z_{\alpha}^{i}}\right], \quad i<j, i, j=1, \ldots, n+1,
$$

which are the rotations and

$$
X_{i}=2 \sum_{\alpha=1}^{n+1}\left[z_{\alpha}^{i} \partial_{z_{\alpha}^{0}}+z_{\alpha}^{0} \partial_{z_{\alpha}^{i}}\right], \quad i=1, \ldots, n+1,
$$

which are the boosts.

Remark that the generators of the group action on $\mathscr{E}_{n+1}$ are found by projection of the generators $X_{i j}$ and $X_{i}$ along the generators of the null cones taken over $\sigma$. The projection along the generators of the null cone may be seen as the projection along the $z_{\alpha}^{0}$-axis followed by a radial projection on the tangent planes to the $n$ spheres. The generators $X_{i j}$ for $i, j=1, \ldots, n$ are the usual rotations in the layers of $\mathscr{E}_{n+1}$. The generators $X_{n+1, i}$ for $i=1, \ldots, n$ are the translations. The generators $X_{i}$ for $i=1, \ldots, n$ are the special conformal transformations and $X_{n+1}$ are the dilatations. The generators $X_{i j}$ are lying in the plane $P$ and are tangent to $\sigma$.

Now, consider the fundamental vector fields over the subspace $\mathcal{N}^{+}$at the section $z_{\alpha}^{0}=1$ defined by $\sigma$. The vector fields $X_{i j}$ are tangent to $\sigma$ by construction. We will construct the projection of the fields $X_{i}$. Denote the projection by $\pi$. 
At $\sigma$, we find

$$
\left.\pi_{P, *} X_{i}\right|_{\sigma}=\sum_{\alpha=1}^{n+1} \partial_{z_{\alpha}^{i}},
$$

where $\pi_{P}$ is the projection on the $P$ plane.

Projection on the tangent plane to $\sigma$ is obtained from the decomposition in the radial component and the tangential component of the vector. Let

$$
\partial_{z_{\alpha}^{i}}=a_{i} \sum_{j=1}^{n+1} z_{\alpha}^{i} \partial_{z_{\alpha}^{i}}+\sum_{k=1}^{n} b_{i k}\left(z_{\alpha}^{n+1} \partial_{z_{\alpha}^{k}}-z_{\alpha}^{k} \partial_{z_{\alpha}^{n+1}}\right) .
$$

For fixed $i$, we find the system

$$
\begin{gathered}
a_{i} z_{\alpha}^{n+1}-\sum_{k=1}^{n} b_{i k} z_{\alpha}^{k}=0, \\
a_{i} z_{\alpha}^{j}+b_{i j} z_{\alpha}^{n+1}=0 \\
a_{i} z_{\alpha}^{i}+b_{i i} z_{\alpha}^{n+1}=1,
\end{gathered}
$$

with $j \neq i$ in the second row. Substitution of the second and third row into the first one gives

$$
a_{i} z_{\alpha}^{n+1}+\sum_{j=1}^{n} \frac{a_{i} z_{\alpha}^{j}}{z_{\alpha}^{n+1}} z_{\alpha}^{j}-\frac{z_{\alpha}^{i}}{z_{\alpha}^{n+1}}=0,
$$

which is equivalent with

$$
a_{i}\left[\left(z_{\alpha}^{n+1}\right)^{2}+\sum_{k=1}^{n}\left(z_{\alpha}^{k}\right)^{2}\right]-z_{\alpha}^{i}=0 .
$$

Hence,

$$
a_{i}=-\frac{z_{\alpha}^{i}}{\sum_{k=1}^{n+1}\left(z_{\alpha}^{k}\right)^{2}} .
$$

Because on $\sigma$ we have $\sum_{i=1}^{n+1} z_{\alpha}^{i} z_{\alpha}^{i}=1$, we find $a_{i}=-z_{\alpha}^{i}$ and $b_{i j}=z_{\alpha}^{i} z_{\alpha}^{j} / z_{\alpha}^{n+1}$ for $j \neq i, b_{i i}=1+z_{\alpha}^{i} z_{\alpha}^{i} / z_{\alpha}^{n+1}$.

The projected vector fields are

$$
\left.\pi_{*} X_{i}\right|_{\sigma}=\sum_{\alpha} \sum_{k=1}^{n} b_{i k}\left[z_{\alpha}^{n+1} \partial_{z_{\alpha}^{k}}-z_{\alpha}^{k} \partial_{z_{\alpha}^{n+1}}\right],
$$

which become

$$
\left.\pi_{*} X_{i}\right|_{\sigma}=\sum_{\alpha}\left[\sum_{k=1}^{n+1} \frac{z_{\alpha}^{i} z_{\alpha}^{k}}{z_{\alpha}^{n+1}}\left[z_{\alpha}^{n+1} \partial_{z_{\alpha}^{k}}-z_{\alpha}^{k} \partial_{z_{\alpha}^{n+1}}\right]+\frac{1}{z_{\alpha}^{n+1}}\left[z_{\alpha}^{n+1} \partial_{z_{\alpha}^{i}}-z_{\alpha}^{i} \partial_{z_{\alpha}^{n+1}}\right]\right]
$$


or

$$
\left.\pi_{*} X_{i}\right|_{\sigma}=\sum_{\alpha}\left[\partial_{z_{\alpha}^{i}}-2 \frac{z_{\alpha}^{i}}{z_{\alpha}^{n+1}} \partial_{z_{\alpha}^{n+1}}+\sum_{k=1}^{n+1} z_{\alpha}^{i} z_{\alpha}^{k} \partial_{z_{\alpha}^{k}}\right] .
$$

But the following set of vector fields commute with the fundamental fields and are transversal to the orbits in ' $W^{\prime}$ :

$$
\begin{gathered}
Y_{\alpha}=\sum_{i=0}^{n+1} z_{\alpha}^{i} \partial_{z_{\alpha}^{i}}, \quad \alpha=1, \ldots, n+1, \\
Y_{\alpha, \beta}=\sum_{i=0}^{n+1}\left[z_{\alpha}^{i} \partial_{z_{\beta}^{i}}-z_{\beta}^{i} \partial_{z_{\alpha}^{i}}\right], \quad \alpha<\beta, \alpha, \beta=1, \ldots, n+1 .
\end{gathered}
$$

Define the fields

$$
\tilde{Y}_{\alpha \beta}=\frac{1}{2\left(z_{\alpha}, z_{\beta}\right)}\left[Y_{\alpha \beta}+Y_{\alpha}-Y_{\beta}\right]
$$

which are equal to

$$
\tilde{Y}_{\alpha \beta}=\frac{1}{2\left(z_{\alpha}, z_{\beta}\right)} \sum_{i=0}^{n+1}\left(z_{\alpha}^{i}-z_{\beta}^{i}\right)\left(\partial_{z_{\beta}^{i}}+\partial_{z_{\alpha}^{i}}\right) .
$$

Proposition 7.12. The following invariant vector fields, taken over $\sigma$, are tangent to $\sigma$

$$
\tilde{Y}_{\alpha \beta \gamma}=\tilde{Y}_{\alpha \beta}+\tilde{Y}_{\beta \gamma}+\tilde{Y}_{\gamma \alpha}
$$

Proof. It suffices to check that $\tilde{Y}_{\alpha \beta \gamma}$ annihilate the forms $\sum_{i=0}^{n+1} z_{\alpha}^{i} z_{\alpha}^{i}$ over the sets $\sum_{i=0}^{n+1} z_{\alpha}^{i} z_{\alpha}^{i}=0$.

The former vector fields equal

$$
\begin{aligned}
\tilde{Y}_{\alpha \beta \gamma}= & \sum_{i=1}^{n+1}\left[\left(\frac{1}{\phi_{\alpha \beta}}+\frac{1}{\phi_{\gamma \alpha}}\right) z_{\alpha}^{i}-\frac{z_{\beta}^{i}}{\phi_{\alpha \beta}}-\frac{z_{\gamma}^{i}}{\phi_{\gamma \alpha}}\right] \partial_{z_{\alpha}^{i}} \\
& +\sum_{i=1}^{n+1}\left[\left(\frac{1}{\phi_{\beta \gamma}}+\frac{1}{\phi_{\alpha \beta}}\right) z_{\beta}^{i}-\frac{z_{\gamma}^{i}}{\phi_{\beta \gamma}}-\frac{z_{\alpha}^{i}}{\phi_{\alpha \beta}}\right] \partial_{z_{\beta}^{i}} \\
& +\sum_{i=1}^{n+1}\left[\left(\frac{1}{\phi_{\gamma \alpha}}+\frac{1}{\phi_{\beta \gamma}}\right) z_{\gamma}^{i}-\frac{z_{\alpha}^{i}}{\phi_{\gamma \alpha}}-\frac{z_{\beta}^{i}}{\phi_{\beta \gamma}}\right] \partial_{z_{\gamma}^{i}} .
\end{aligned}
$$

We omit the proof of following proposition.

Proposition 7.13. The set $\tilde{Y}_{\alpha \beta(\beta+1)}$, with $\alpha<\beta<n$ if $\alpha=1$ and $\alpha<\beta<$ $n+1$ otherwise, span an invariant normal bundle to the orbits of $\mathrm{CO}^{(1)}(n)$ in $\sigma$.

The existence of these vector fields allows the construction of an invariant metric on $\sigma$. We proceed as in the orthogonal case and require the normal 
bundle to be orthogonal to the orbits. In the normal bundle, we introduce the Euclidean metric with respect to the above constructed vector fields, and, in the orbits, we use the Killing form $K$ of the Lie algebra co ${ }^{(1)}(n)$ with respect to the fundamental vector fields. Let $\hat{q}_{2}$ be this metric, and $\tilde{q}_{2}$ the corresponding function in the tangent space.

Consider the space $\mathscr{g}^{(1,0, \ldots, 0) \mathscr{E}_{n+1}}=J^{1} V_{1} \times V_{2} \times \cdots \times V_{n+1}$.

Taking the prolongation of $\mathscr{I}^{(1)}(\mathcal{W})$ on $\pi^{(-1)} W=W^{\prime}$, we, as a consequence of the definition of the set $\mathscr{I}^{(1)}(\mathscr{W})$, find

$$
\operatorname{dim} \mathcal{F}^{(1)}\left(\mathscr{W}^{\prime}\right)=\frac{(n+1)(n-2)}{2}+n+1=\frac{n^{2}+n-4}{2} .
$$

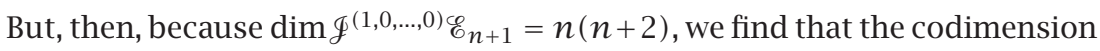
of the orbits in the level surfaces of the sheaf generated by $\Phi^{(1)}\left({ }^{\prime} W^{\prime}\right)$ equals one. The extra invariant generator is given by the layer metric $\tilde{q}_{2}^{1}$, which is nothing but the restriction of the metric $\tilde{q}_{2}$ to the first-layer tangent bundle.

This brings us to the following theorem.

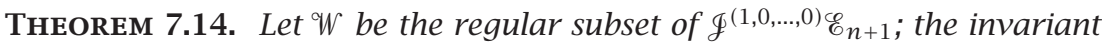
sheaf is generated by $\mathscr{I}_{\mathscr{W}}=\mathscr{I}_{\mathscr{W}_{n+1}} \cup \mathscr{I}_{\mathscr{W}_{n+1}}^{(1)} \cup\left\{\tilde{q}_{2}^{1}\right\}$.

The same procedure applies to the higher-order cases because the group $O^{+}(1, n+1)$ extends linearly on the jet bundles over the base manifold which allows to construct the transversal vector fields $\tilde{Y}_{\alpha \beta \gamma}$ in exactly the same way, and only the embedding changes. Let $\sigma: \mathscr{E}_{n+1} \hookrightarrow \mathscr{V}_{n+1}$. Then,

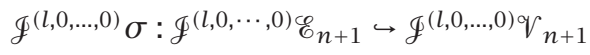

is the natural jet bundle embedding.

\section{REFERENCES}

[1] A. F. Beardon, The Geometry of Discrete Groups, Graduate Texts in Mathematics, vol. 91, Springer-Verlag, New York, 1983.

[2] H. Boerner, Representation of Groups with Special Consideration for the Needs of Modern Physics, North-Holland Publishing, Amsterdam, 1963.

[3] M. H. Brill, E. B. Barrett, and P. M. Payton, Projective invariants for curves in two and three dimensions, Geometric Invariance in Computer Vision (J. L. Mundy and A. Zisserman, eds.), Artificial Intelligence, MIT Press, Massachusetts, 1992, pp. 193-214.

[4] H. S. M Coxeter, The representation of conformal space on a quadric, Ann. of Math. 37 (1936), 416-426.

[5] P. F. Dhooghe, Contact transformations, contact algebras and lifts on a jet bundle, Ann. Mat. Pura Appl. (4) 131 (1982), 291-309.

[6] _ On projective invariants of curves, Results Math. 27 (1995), no. 3-4, 237243.

[7] _ Multi local invariants on real unit balls, Math. J. Okayama Univ. 41 (1999), 149-179.

[8] M. Fels and P. J. Olver, Moving coframes. I. A practical algorithm, Acta Appl. Math. 51 (1998), no. 2, 161-213. 
[9] W. Fulton and J. Harris, Representation Theory, Graduate Texts in Mathematics, vol. 129, Springer-Verlag, New York, 1991.

[10] V. W. Guillemin and S. Sternberg, An algebraic model of transitive differential geometry, Bull. Amer. Math. Soc. 70 (1964), 16-47.

[11] S. Helgason, Differential Geometry, Lie Groups, and Symmetric Spaces, Pure and Applied Mathematics, vol. 80, Academic Press, New York, 1978.

[12] R. Howe, Remarks on classical invariant theory, Trans. Amer. Math. Soc. 313 (1989), no. 2, 539-570.

[13] S. Kobayashi and K. Nomizu, Foundations of Differential Geometry. Vol I, Interscience Publishers, New York, 1963.

[14] I. S. Krasil'shchik, V. V. Lychagin, and A. M. Vinogradov, Geometry of Jet Spaces and Nonlinear Partial Differential Equations, Advanced Studies in Contemporary Mathematics, vol. 1, Gordon and Breach Science Publishers, New York, 1986.

[15] P. J. Olver, Applications of Lie Groups to Differential Equations, Graduate Texts in Mathematics, vol. 107, Springer-Verlag, New York, 1993.

[16]_ Differential invariants, Acta Appl. Math. 41 (1995), no. 1-3, 271-284.

[17] _ Moving frames and joint differential invariants, Regul. Chaotic Dyn. 4 (1999), no. 4, 3-18.

[18]__ Geometric foundations of numerical algorithms and symmetry, Appl. Algebra Engrg. Comm. Comput. 11 (2001), no. 5, 417-436.

[19]___ Joint invariant signatures, Found. Comput. Math. 1 (2001), no. 1, 3-67.

[20] P. J. Olver, G. Sapiro, and A. Tannenbaum, Differential invariant signatures and flows in computer vision: a symmetry group approach, Geometry Driven Diffusion in Computer Vision (B. M. ter Haar Romeny, ed.), Kluwer Academic Publishers, Dordrecht, 1994.

[21] T. Sasaki, Projective differential geometry and linear homogeneous differential equations, preprint, 1989.

[22] S. Sternberg, Lectures on Differential Geometry, Prentice-Hall, New Jersey, 1965.

[23] T. Umeda, The Capelli identities, a century after, Selected Papers on Harmonic Analysis, Groups, and Invariants, Amer. Math. Soc. Transl. Ser. 2, vol. 183, American Mathematical Society, Rhode Island, 1998, pp. 51-78.

[24] L. J. Van Gool, T. Moons, E. Pauwels, and A. Oosterlinck, Semi-differential invariants, Geometric Invariance in Computer Vision (J. L. Mundy and A. Zisserman, eds.), Artificial Intelligence, MIT Press, Massachusetts, 1992, pp. 157192.

[25] H. Weyl, The Classical Groups, Princeton University Press, New Jersey, 1946.

[26] E. Wilczynski, Projective Differential Geometry of Curves and Ruled Surfaces, Teubner, Leibzig, 1906.

Paul F. Dhooghe: Department of Mathematics, Center for Pure and Applied Differential Geometry, KULeuven, Celestijnenlaan 200 B, 3001 Leuven, Belgium

E-mail address: Pau1 . Dhooghe@wi s. ku1 euven. ac . be 


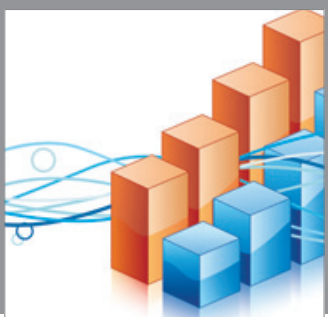

Advances in

Operations Research

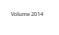

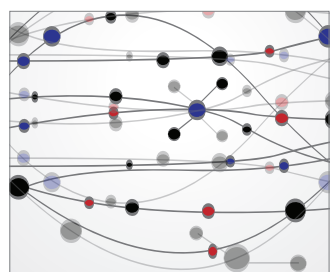

\section{The Scientific} World Journal
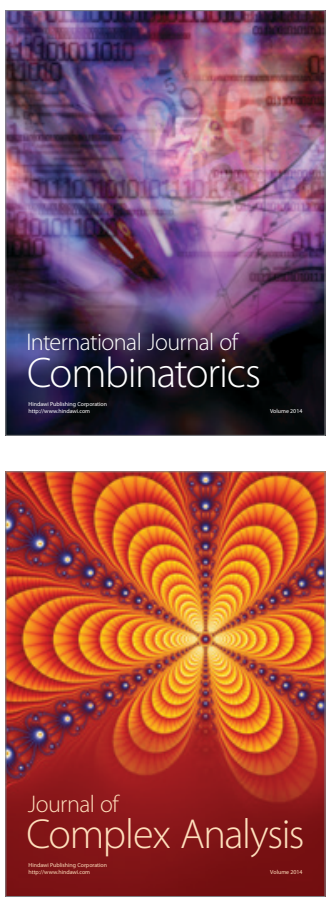

International Journal of

Mathematics and

Mathematical

Sciences
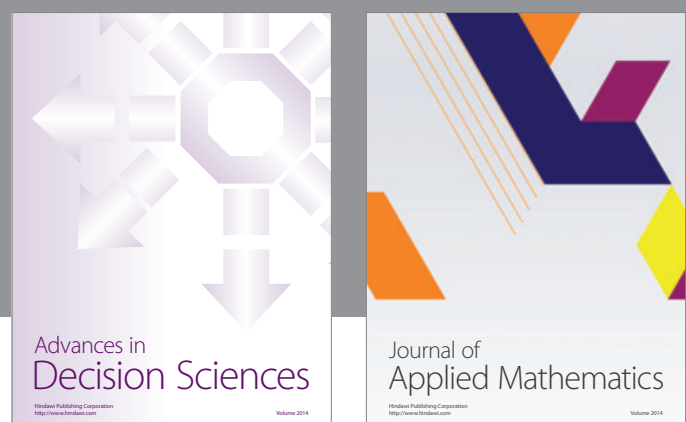

Journal of

Applied Mathematics
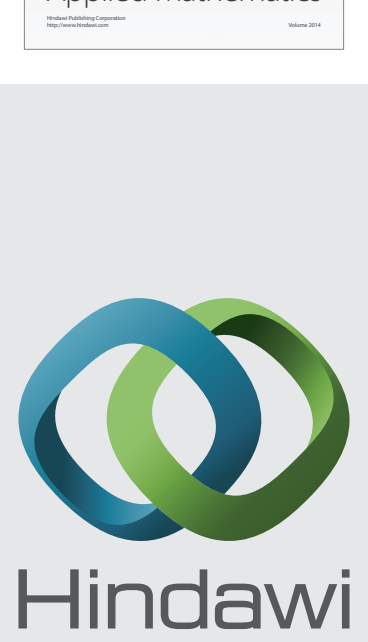

Submit your manuscripts at http://www.hindawi.com
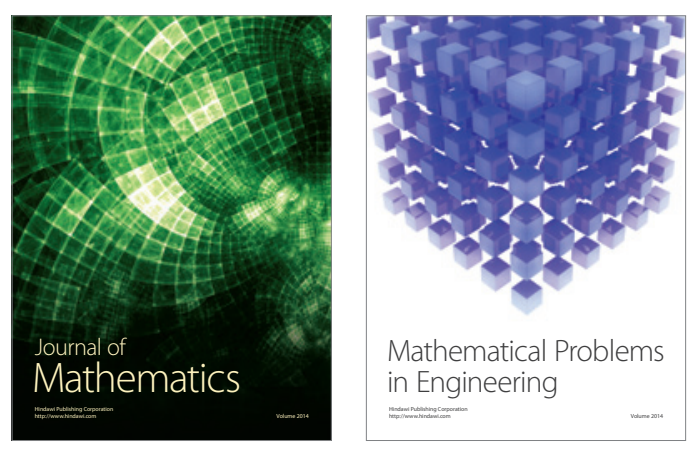

Mathematical Problems in Engineering
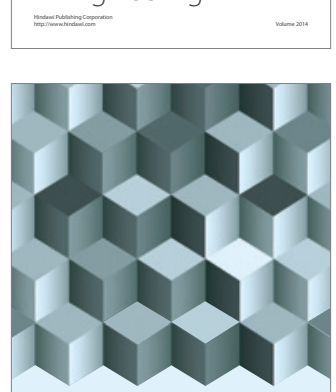

Journal of

Function Spaces
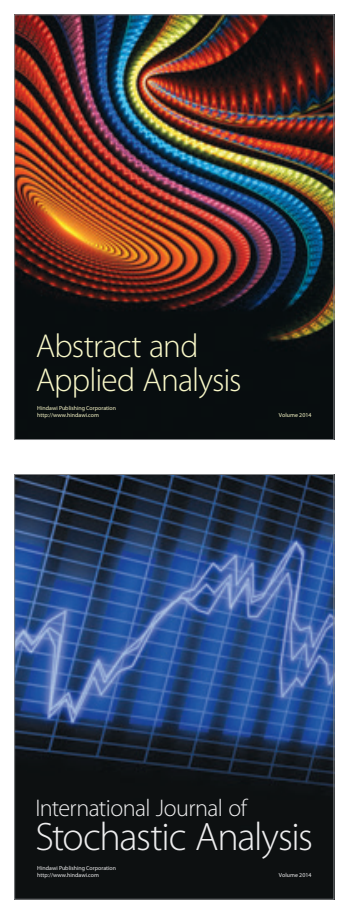

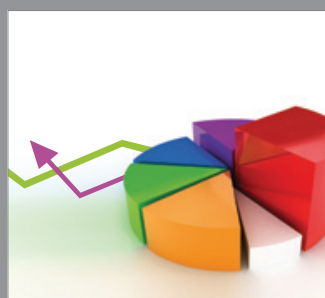

ournal of

Probability and Statistics

Promensencen
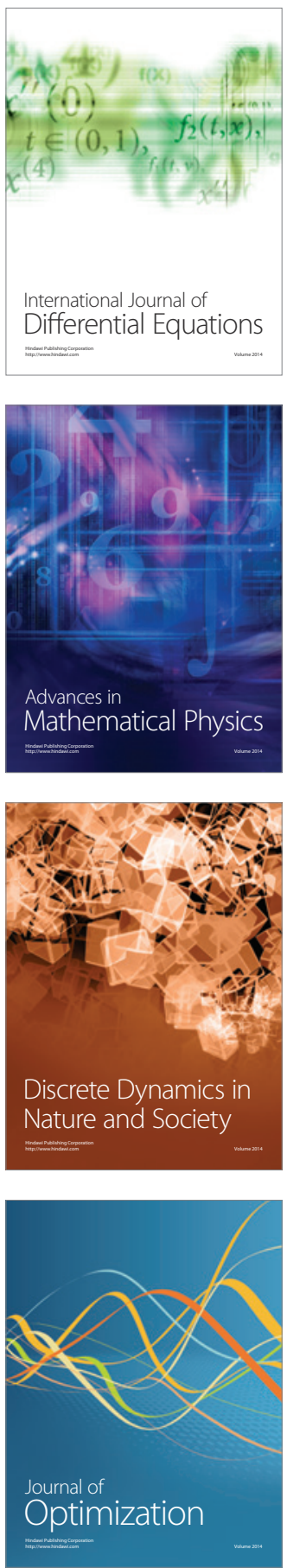